\title{
Evolutionary dynamics of zero-sum games ${ }^{\star}$
}

\author{
Ethan Akin ${ }^{1}$ and Viktor Losert ${ }^{2}$ \\ ${ }^{1}$ Mathematics Department, The City College, 137 St. and Convent Ave., New York, NY 10031, \\ USA. \\ ${ }^{2}$ Institut für Mathematik, Universität Wien, Strudlhofgasse 4, A-1090 Wien, Austria.
}

\begin{abstract}
Aim model in terms of differential equations is used to explain mammalian ovulation control, in particular regulation for a prescribed number of mature eggs.
\end{abstract}

Key words: Ovulation — regulation of egg number

\section{Introduction}

A two-player symmetric game consists of a finite set of strategies indexed by $I=\{1, \ldots, n\}$ and an $n \times n$ payoff matrix $\left(a_{i j}\right)$. When an $i$ player meets a $j$ player their payoffs are $a_{i j}$ and $a_{j i}$, respectively.

In evolutionary game dynamics we imagine a large population of game players, each with a fixed strategy. The state of the population is a vector in $\mathbb{R}_{+}^{n}=$ $\left\{x \in \mathbb{R}^{n}: x_{i} \geqslant 0\right\}$ where $x_{i}$ measures the subpopulation of $i$ strategists. So the total population size is $|x|=\sum_{i} x_{i}$. The associated distribution vector lies in the simplex $\Delta=\left\{p \in \mathbb{R}_{+}^{n}: \sum_{i} p_{i}=1\right\}$ where $p_{i}=x_{i} /|x|$, the ratio of $i$ strategists to the total population. The dynamics comes from assuming that payoff is measured in units of fitness, i.e. relative growth rate, and is added to some background growth rate. Thus, we assume the system of differential equations:

$$
\frac{d x_{i}}{d t}=x_{i}\left(r+a_{i p}\right)
$$

where $r$ is the background, strategy-independent growth rate and $a_{i p} \equiv \sum_{j} p_{j} a_{i j}$ is the average payoff to the $i$ th strategist because his opponent is a $j$ strategist with probability $p_{j}$.

Summing on $i$, and using $x_{i}=|x| p_{i}$ we get the equation for the growth of the total population size:

where $a_{p p}=\sum_{i} p_{i} a_{i p}=\sum_{i, j} p_{i} p_{j} a_{i j}$.

$$
\frac{d|x|}{d t}=|x|\left(r+a_{p p}\right)
$$

Using $d \ln p_{i}=d \ln x_{i}-d \ln |x|$ we obtain from $(0.1)$ and $(0.2)$ the system of differential equations describing the evolution of the distribution vector:

$$
\frac{d p_{i}}{d t}=p_{i}\left(a_{i p}-a_{p p}\right)
$$


Thus, the relative rate of increase of the frequency of $i$ players is given by the average excess in payoff between an $i$ player and a random member of the population.

Notice that the background rate $r$ need not be a constant. It can depend on the population size $|x|$ or even on the entire state vector $x$. But as long as its effect on fitness is additive, it cancels out in (0.3) and so we obtain a dynamical system on the simplex which depends only upon the payoff matrix. When it is solved, the solution can be substituted back into $(0.2)$ to get a single timedependent equation in population size.

Because the simplex $\Delta$ is compact, we can display the solution of $(0.3)$ as a smooth map $\Phi: \Delta \times \mathbb{R} \rightarrow \Delta$ called the flow of the system. With $p \in \Delta$ fixed the function of $t: \Phi(p, t)$ is the solution path for the system with initial point $p$. With $t$ fixed we obtain a smooth map $\Phi^{t}: \Delta \rightarrow \Delta$ and the family of mappings satisfies the group properties: $\Phi^{0}=$ identity and $\Phi^{t} \circ \Phi^{s}=\Phi^{t+s}$. In particular, each $\Phi^{t}$ is a diffeomorphism, i.e. it has a smooth inverse, namely $\Phi^{-t}$. See, e.g. Abraham and Marsden (1978) Section 2.1.

Suppose we attempt to solve system (0.1) numerically using the simplest approach, namely Euler's polygonal approximation method. This amounts to replacing the derivative $d x_{i} / d t$ by the difference quotient $\delta x_{i} / \tau$ with $\tau>0$ a fixed, small increment, i.e.

$$
\delta x_{i}=x_{i} \tau\left(r+a_{i p}\right)
$$

or alternatively after one step $x$ is mapped to the vector $x^{\prime}$ with

$$
x_{i}^{\prime}=x_{i}\left[1+\tau\left(r+a_{i p}\right)\right] .
$$

So the total population size changes by:

$$
\left|x^{\prime}\right|=|x|\left[1+\tau\left(r+a_{p p}\right)\right] \text {. }
$$

Because $p_{i}^{\prime}=x_{i}^{\prime} /\left|x^{\prime}\right|$ and $p_{i}=x_{i} /|x|$ we get:

$$
p_{i}^{\prime}=p_{i}\left[1+\tau\left(r+a_{i p}\right)\right] /\left[1+\tau\left(r+a_{p p}\right)\right] .
$$

Ignoring the fact that $r$ may not be constant we define $\tilde{\tau}=\tau /(1+\tau r)$ and so the mapping from $p$ to $p^{\prime}$ can be written as $p^{\prime}=F_{\tilde{\tau}}(p)$ where $F_{\tilde{\tau}}: \Delta \rightarrow \Delta$ is defined by:

$$
F_{\tilde{\tau}}(p)_{i}=p_{i}\left(1+\tilde{\tau} a_{i p}\right) /\left(1+\tilde{\tau} a_{p p}\right) .
$$

Euler's result that the polygonal solutions approach the solution of the differential equation says that for any $p$ in $\Delta$ and $t>0$ :

$$
\lim \left[F_{\tilde{\tau}}\right]^{n}(p)=\Phi^{t}(p)
$$

where $n \rightarrow \infty$ and $\tilde{\tau} \rightarrow 0$ so balanced that the product $n \tilde{\tau} \rightarrow t$. Here the exponent $n$ represents $n$-fold iteration of the map $F_{\tilde{\tau}}$ so $n$ is a whole number. See, e.g. Abraham and Marsden (1978) Theorem 2.1.26.

This result motivates the hope that the dynamics of the continuous time system (0.3) might be similar to the dynamics of the discrete time system:

$$
\delta p_{i}=p_{i}\left(a_{i p}-a_{p p}\right) /\left(1+a_{p p}\right)
$$


which is just a rewriting of (0.4) with $\tilde{\tau}=1$, i.e. we consider the map $F: \Delta \rightarrow \Delta$ defined by

$$
F(p)_{i}=p_{i}\left(1+a_{i p}\right) /\left(1+a_{p p}\right) .
$$

Since we have, in effect, subsumed $\tilde{\tau}$ into the matrix we may need conditions that the entries $a_{i j}$ be small. The only conditions of this sort that we will always impose are those necessary that $F(p)_{i}$ be defined and nonnegative:

$$
1+a_{i j} \geqslant 0 \text { and } 1+a_{i i}>0 \text { for } i, j=1, \ldots, n \text {. }
$$

i.e. the matrix $1+a_{i j}$ is nonnegative with a strictly positive diagonal. These conditions ensure that $1+a_{i p} \geqslant p_{i}\left(1+a_{i i}\right)$ and so $1+a_{i p}>0$ if $p_{i}>0$. In particular, $1+a_{p p}>0$ for all $p$ in $\Delta$.

In the case where the matrix $a_{i j}$ is itself symmetric, i.e. $a_{i j}=a_{j i}$, these systems have been widely studied as two versions of the one-locus- $n$-allele model of classical population genetics, $(0.5)$ and $(0.6)$ due to Wright and $(0.3)$ due to Fisher. Here the phrase weak selection (i.e. $\left|a_{i j}\right|$ small) has been used as the label on the bridge to cross from the biologically more reasonable discrete time system to the mathematically more tractable differential equation. As discussed in Losert and Akin (1983) the hope that the two systems will behave similarly seems well justified in this symmetric case.

The general system of equations (0.3) was introduced by Taylor and Jonker (1978) as we described above as a dynamic model for Maynard Smith's applications of game theory to evolution (see Maynard Smith (1982) for a survey). Independently, this system of equations was introduced by Eigen's group studying origin of life problems (see Eigen and Schuster (1979) for a survey). The discrete time model (0.5) is due to Hines (1980) and to Losert and Akin (1983). In particular, in the latter paper is proved the following result which we will use repeatedly:

Theorem 1. Assuming conditions (0.7) the map $F: \Delta \rightarrow \Delta$ defined by (0.6) is a homeomorphism, i.e. $F$ is one-to-one and onto and the inverse function $F^{-1}$ is continuous. $F^{-1}$ is differentiable in the interior of $\Delta$ and if $1+a_{i j}>0$ for all $i, j$ then $F^{-1}: \Delta \rightarrow \Delta$ is a smooth map, i.e. $F$ is a diffeomorphism.

In this paper we will examine the case antipodal to the population genetic model, namely where the payoff matrix is antisymmetric. Since this condition can be written $a_{i j}+a_{j i}=0$ such games are called zero-sum.

In addition to its interest as a special case of evolutionary game models this class of systems has a separate pattern of applications all its own. Nagylaki $(1983 \mathrm{a}, \mathrm{b})$ has introduced (0.6) with antisymmetric $a_{i j}$ as a model for gene conversion. We would like to express our gratitude to Professor Nagylaki for our introduction to the problem, for many helpful discussions and, above all, for his emphasis on the radical difference between the discrete and continuous models in the antisymmetric case.

In Sect. 1 we use a common pattern of assaying the behavior of certain $\log$-linear functions on orbits to provide an overview of the behavior of the two kinds of models. The distinct behaviors thus revealed are examined in detail in the later sections. In Sect. 2 we use techniques of Conley and Smale to construct 
a closed set to which - we believe - almost all orbits of the discrete system are attracted. In Sect. 3 we show that the continuous system exhibits the conservative behavior of classical Hamiltonian dynamics.

A related class of difference equations appears in Hofbauer (1984).

\section{Classification and general behavior}

From now on we will assume the payoff matrix is antisymmetric, i.e. $a_{i j}=-a_{j i}$. This implies $a_{p p}=\sum_{i, j} p_{i} p_{j} a_{i j}=0$ for any point $p$ and so the associated system of differential equations on $\Delta,(0.3)$, becomes:

$$
\frac{d p_{i}}{d t}=p_{i} a_{i p}
$$

where $a_{i p}=\sum_{j} p_{j} a_{i j}$.

Whenever we consider the discrete time system we will assume conditions (0.7) which in the antisymmetric case become:

$$
\left|a_{i j}\right| \leqslant 1 \text { for all } i, j \text {. }
$$

The discrete time system $(0.5)$ becomes:

$$
\delta p_{i}=p_{i} a_{i p}
$$

Equivalently, the discrete dynamic is given by the mapping $F: \Delta \rightarrow \Delta$ with

$$
F(p)_{i}=p_{i}\left(1+a_{i p}\right) \text {. }
$$

By Theorem 1 of the introduction, $F$ is a homeomorphism and if the inequalities of (1.2) are all strict $F$ is a diffeomorphism. Recall that integrating (1.1) yields the flow $\Phi: \Delta \times \mathbb{R} \rightarrow \Delta$ which displays all the solution paths at once. Because $F$ of (1.4) is invertible, we can define the discrete time flow $F: \Delta \times \mathbb{Z} \rightarrow \Delta$ where $\mathbb{Z}$ is the set of integers. We use the same symbol $F$ for map and flow because when by analogy with $\Phi^{t}: \Delta \rightarrow \Delta$ we look at $F^{t}: \Delta \rightarrow \Delta$ this map is just $F$ iterated $t$ times when $t$ is a positive integer and is $F^{-1}$ iterated $|t|$ times when $t$ is a negative integer. $F^{0}$ is the identity map. Thus, if $p \in \Delta, \Phi^{t}(p)$ as a function of $t$ in $\mathbb{R}$ is the solution path of (1.1) with initial condition $p$. Similarly, $F^{t}(p)$ as a function of $t$ in $\mathbb{Z}$ is the solution path of (1.3) with initial condition $p$.

A point $e$ of $\Delta$ is called an equilibrium if the solution path remains constant at $e$ as $t$ varies. The conditions that $e$ be an equilibrium for (1.1) or (1.3) are $d p_{i} / d t=0$ for all $i$ or $\delta p_{i}=0$ for all $i$ respectively. So the two systems have the same set of equilibria: $e$ is an equilibrium if for all $i$ either $e_{i}=0$ or $a_{i e}=0$ (or both).

Introducing the concept of support, we can restate the equilibrium conditions. If $x$ is a vector of $\mathbb{R}^{n}$ then the support of $x$, denoted $\operatorname{supp}(x)$, is $\left\{i \in I: x_{i} \neq 0\right\}$. In particular, if $p \in \Delta$ then $p_{i}>0$ for $i \in \operatorname{supp}(p)$ and $p_{i}=0$ for $i \notin \operatorname{supp}(p)$. If $J \subset I$ we define

$$
\begin{aligned}
& \mathbb{R}^{J}=\left\{x \in \mathbb{R}^{n}: \operatorname{supp}(x) \subset J\right\}=\left\{x \in \mathbb{R}^{n}: x_{i}=0 \text { for } i \notin J\right\} . \\
& \mathbb{R}_{+}^{J}=\mathbb{R}_{+}^{n} \cap \mathbb{R}^{J}, \quad \Delta^{J}=\Delta \cap \mathbb{R}^{J} \\
& \stackrel{\circ}{\mathbb{R}}_{+}^{J}=\left\{x \in \mathbb{R}_{+}^{n}: \operatorname{supp}(x)=J\right\}=\left\{x \in \mathbb{R}^{J}: x_{i}>0 \text { for } i \in J\right\} \\
& \stackrel{\circ}{J}^{J}=\Delta \cap \mathbb{R}_{+}^{J}=\{p \in \Delta: \operatorname{supp}(p)=J\} .
\end{aligned}
$$


In particular, the set of interior vectors of $\Delta$, denoted $\dot{\Delta}$, consists of the positive vectors of $\Delta$, i.e. $\Delta=\left\{p \in \Delta: p_{i}>0\right.$ for all $\left.i\right\}$. The remaining points of $\Delta$ form the boundary of $\Delta, \partial \Delta=\Delta-\Delta=\left\{p \in \Delta: p_{i}=0\right.$ for some $\left.i\right\}$.

Thus, $e \in \Delta$ is an equilibrium if $a_{i e}$ vanishes for every $i$ in the support of $e$. In particular, $e$ is an interior equilibrium if $e \in \Delta$ and $a_{i e}=0$ for all $i$. At the other extreme each vertex of the simplex is an equilibrium. This follows from the general fact that the support remains constant on every solution path, i.e. each $\Delta^{J}$ is an invariant set.

When $p$ is not an equilibrium we are interested in describing its asymptotic behavior, e.g. computing the limit as $t \rightarrow \infty$ of $\Phi^{t}(p)$ or $F^{t}(p)$. However, these functions of $t$ need not tend to a unique limit point as $t \rightarrow \infty$. So instead we consider $\omega(p)$ defined to be the set of limits of all convergent subsequences $\Phi^{t_{n}}(p)$ or $F^{t_{n}}(p)$ where $t_{n}$ is a sequence of values in $\mathbb{R}$ or $\mathbb{Z}$ approaching $\infty$. An exercise in topology yields the equivalent definition

$$
\begin{aligned}
& \omega_{\Phi}(p)=\bigcap_{t} \overline{\bigcup_{s \geqslant t}\left\{\Phi^{s}(p)\right\}} \text { for (1.1) } \\
& \omega_{F}(p)=\bigcap_{t} \overline{\bigcup_{s \geqslant t}\left\{F^{s}(p)\right\}} \text {, for }(1.3),
\end{aligned}
$$

where $s, t$ vary over $\mathbb{R}$ for (1.1) and over $\mathbb{Z}$ for (1.3). The bar denotes the closure operation in $\Delta$. As the decreasing intersection of compact sets $\omega(p)$ is nonempty in each case.

Instead of asking where $p$ is going we can ask where it is coming from and define $\alpha(p)$ to be the set of limits of convergent subsequences where $t_{n} \rightarrow \infty$.

$$
\begin{array}{ll}
\alpha_{\Phi}(p)=\bigcap_{t} \overline{\bigcup_{s \leqslant t}\left\{\Phi^{s}(p)\right\}} & \text { for (1.1) } \\
\alpha_{F}(p)=\bigcap_{t} \overline{\bigcup_{s \leqslant t}\left\{F^{s}(p)\right\}} & \text { for }(1.3) .
\end{array}
$$

For any point $p, \omega(p)$ and $\alpha(p)$ are nonempty compact sets which are invariant under the corresponding dynamic, e.g. if $q \in \omega_{\Phi}(p)$ then the entire solution path $\Phi^{t}(q)$ remains in $\omega_{\Phi}(p)$. In particular, if any of these four sets consists of a single point, i.e. the corresponding limit exists, then that point is an equilibrium. If $e$ is an equilibrium then each of the four sets consists of $e$ itself.

To illustrate these concepts and to introduce the reader to the difference between the discrete and continuous time models we begin with the classic example of a zero sum game, paper-rock-scissors. Here $n=3$ and the matrix is:

$$
\left(\begin{array}{rrr}
0 & \tau & -\tau \\
-\tau & 0 & \tau \\
\tau & -\tau & 0
\end{array}\right) \quad 0<\tau<1
$$

So $a_{i p}=\tau\left(p_{i+1}-p_{i-1}\right)$ where the arithmetic in the indices is modulo 3 . As shown in Fig. 1 the only equilibrium other than the three vertices is the center $e$ with $e_{i}=\frac{1}{3}$. If we define the symmetric function $\pi(p)=p_{1} p_{2} p_{3}$ on $\Delta$ it is easy to check using (1.1) that $d \pi / d t=0$ at every point $p$. Thus, $\pi$ remains constant on the solution paths $\Phi^{t}(p)$ for the differential equation. $\pi=0$ on the boundary of $\Delta$ and $\pi(e)=\frac{1}{27}$. If $0<c<\frac{1}{27}$ then the set $\{p: \pi(p)=c\}$ is a closed curve about $e$ 


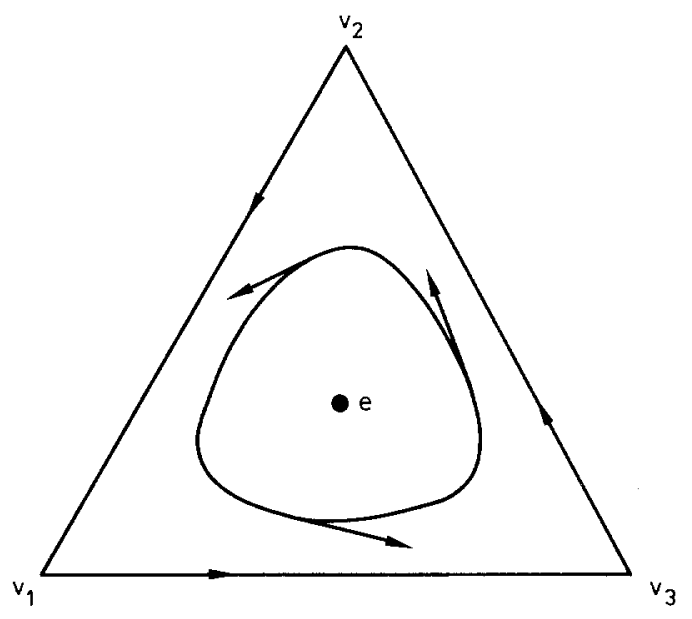

Fig. 1

which is invariant under the flow. If $p \neq e$ is an interior point then the solution $\Phi^{t}(p)$ is periodic, cycling around the curve with $c=\pi(p)$. In particular, $\alpha_{\Phi}(p)=$ $\omega_{\Phi}(p)$ consists of the entire curve. The equilibrium $e$ is stable but not asymptotically stable, i.e. if $p$ is near $e$ then the solution path $\Phi^{t}(p)$ remains near but does not approach $e$.

The invariance of the convex curve $\{\pi=c\}$ means that, as shown in Fig. 1, the vector field associated with (1.1) is everywhere tangent to the curve. Now if we look at the vector based at $p \neq e, F(p)$ is exactly the tip of the arrow, which lies outside the curve, i.e. it is closer to the boundary than $p$ and so $\pi(F(p))<$ $\pi(p)$. Thus, if $p$ is any nonequilibrium interior point the sequence of values $\pi\left(F^{t}(p)\right)$ is a monotone decreasing function of $t$ as $t$ varies in $\mathbb{Z}$. As we traverse the orbit backwards in time $F^{t}(p)$ spirals inward toward $e$, i.e. $\alpha_{F}(p)=e$. As we move forward in time $F^{t}(p)$ spirals outward toward the boundary but does not approach any limiting equilibrium. Instead $\omega_{F}(p)$ is a closed invariant subset of the boundary containing all three vertices. When $t$ is large $F^{t}(p)$ is close to the boundary and appears to cycle around it. This cycling behavior of points near the boundary contrasts with the behavior of points actually on the boundary all of which approach some vertex equilibrium. Thus, the boundary is a closed invariant set attracting all nonequilibrium interior solution paths for (1.3) but is unlike a limit cycle in that points near the set do not behave like points on the set itself.

The analysis of the rest of this section is based on two ideas. The first is to hunt for functions like $\pi$. In practice it is more convenient to take the logarithm and define for $b \in \mathbb{R}^{n}$ :

$$
L^{b}(p)=\sum b_{i} \ln p_{i}, \quad \operatorname{supp}(p) \supset \operatorname{supp}(b) .
$$

Here the sum is taken over all $i$ in the support of $b$. In order that the $\ln p_{i}$ be finite for all such $i, p_{i}$ must be positive for $i \in \operatorname{supp}(b)$. Thus, $L^{b}$ is defined and smooth on $\{p: \operatorname{supp}(p) \supset \operatorname{supp}(b)\}$, an open subset of $\Delta$ containing $\check{\Delta}$.

We repeatedly use the strict concavity of the log function. For example, with $q \in \Delta$ we define

$$
I^{q}(p)=-\sum q_{i} \ln \left(p_{i} / q_{i}\right), \quad \operatorname{supp}(p) \supset \operatorname{supp}(q)
$$


which differs from $-L^{q}(p)$ by the constant $\sum q_{i} \ln q_{i}$. Concavity implies that $I^{q}$ can be used as a measure of displacement from $q$ because

$$
I^{q}(p) \geqslant-\ln \sum q_{i}\left(p_{i} / q_{i}\right) \geqslant-\ln 1=0
$$

with equality only when the ratio $p_{i} / q_{i}$ is constantly 1 for all $i$ in $\operatorname{supp}(q)$, i.e. when $p=q$. Thus, $I^{q}$ is a nonnegative function vanishing only at $q$.

The following Lemma describes the vectors $b$ for which $L^{b}$ will prove useful.

1. Lemma. (a) Let $b \in \mathbb{R}^{n}$ such that $a_{b j} \leqslant 0$ for all $j$. Assume the continuous dynamic (1.1) on $\Delta$. At any point $p$ with $\operatorname{supp}(p) \supset \operatorname{supp}(b)$

$$
\frac{d L^{b}}{d t} \leqslant 0
$$

with strict inequality unless $a_{b j}=0$ for all $j$ in $\operatorname{supp}(p)$.

(b) Let $b \in \mathbb{R}_{+}^{n}$ such that $a_{b j} \leqslant 0$ for all $j$. Assume the discrete time dynamic (1.3). At any point $p$ with $\operatorname{supp}(p) \supset \operatorname{supp}(b)$

$$
\delta L^{b} \leqslant 0
$$

with strict inequality unless $a_{i p}=0$ for all $i$ in $\operatorname{supp}(b)$.

Proof. For (a) we have the easy direct computation

$$
\frac{d L^{b}}{d t}=\sum b_{j} \frac{d \ln }{d t} p_{j}=a_{b p}
$$

As $a_{b j} \leqslant 0$ for all $j$ the average $a_{b p} \leqslant 0$ and $a_{b j}<0$ for any $j$ in $\operatorname{supp}(p)$ implies $a_{b p}<0$.

Excluding the trivial case $b=0$, we can multiply $b \in \mathbb{R}_{+}^{n}$ by a positive constant to assume $\sum b_{j}=1$, i.e. $b \in \Delta$. Then for (b) concavity of the log function implies:

$$
\begin{aligned}
\delta L^{b}(p) & =L^{b}(F(p))-L^{b}(p)=\sum b_{j} \ln \left(F(p)_{j} / p_{j}\right) \\
& =\sum b_{j} \ln \left(1+a_{j p}\right) \leqslant \ln \left(1+a_{b p}\right) \leqslant \ln 1=0 .
\end{aligned}
$$

The first inequality is strict unless all $a_{j p}$ 's are equal for $j$ in $\operatorname{supp}(b)$ and the second is strict unless $a_{b p}=0$. Thus, $\delta L^{b} \leqslant 0$ and the inequality is strict unless $a_{j p}=0$ for all $j$ in $\operatorname{supp}(b)$.

Q.E.D.

Remark: We will see that the difference in behavior between (1.1) and (1.3) is derived from the difference in conditions for strictness of the inequality. Suppose $b \in \mathbb{R}_{+}^{n}$ and $a_{b j}=0$ for all $j$. Then $L^{b}$ remains constant on the solution path of (1.1) through $p$ because $a_{b p}=0$. But $L^{b}$ will still be strictly decreasing on the solution path of (1.3) through $p$ unless, in addition, $a_{i p}=0$ for all $i$ in $\operatorname{supp}(b)$.

The second major idea is the use of separation theorems for polyhedral sets. Define:

$$
\begin{aligned}
& E_{0}=\left\{e \in \Delta: a_{i e}=0 \text { for all } i\right\} \\
& E_{+}=\left\{e \in \Delta: a_{i e} \geqslant 0 \text { for all } i, \text { with at least one inequality strict }\right\} . \\
& E_{-}=\left\{e \in \Delta: a_{i e} \leqslant 0 \text { for all } i, \text { with at least one inequality strict }\right\} .
\end{aligned}
$$


2. Theorem. $E_{0}, E_{+}$and $E_{-}$are convex subsets of $\Delta$ consisting entirely of equilibria. $E_{0}$ is exactly the set of interior equilibria. $E_{+}$and $E_{-}$are subsets of the boundary.

For any antisymmetric matrix $a_{i j}$ exactly one of the following two cases holds:

Interior equilibrium case: $E_{0}$ is nonempty and $E_{+}$and $E_{-}$are both empty sets.

No interior equilibrium case: $E_{0}$ is empty and both $E_{+}$and $E_{-}$are nonempty.

Proof: ${ }^{1}$ By definition $E_{0}$ consists of all interior equilibria. Convexity of the three sets is clear. Now suppose $e \in E_{+} .0=a_{e e}=\sum_{i} e_{i} a_{i e}$ and $e_{i} a_{i e} \geqslant 0$ for all $i$ implies $e_{i} a_{i e}=0$ for all $i$. Hence, $a_{i e}=0$ for $i$ in $\operatorname{supp}(e)$, i.e. $e$ is an equilibrium, and $e_{i}=0$ when $a_{i e}>0$, i.e. $e$ lies in the boundary of $\Delta$. Finally, we note that if $e \in E_{+}$

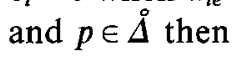

$$
0<\sum p_{i} a_{i e}=a_{p e}=-a_{e p}=-\sum e_{i} a_{i p} .
$$

In particular, $p \notin E_{0}$. So if $E_{+}$is nonempty, $E_{0}$ is empty. Suppose, on the other hand, that $E_{+}$is empty. Define

$$
Y=\left\{y \in \mathbb{R}^{n}: y_{i}=a_{i x} \text { for some } x \text { in } \mathbb{R}_{+}^{n}\right\} .
$$

Thus, $Y$ is the convex cone on the columns of the matrix $a_{i j}$. The hypothesis that $E_{+}=\emptyset$ implies that $Y$ intersects the positive orthant $\mathbb{R}_{+}^{n}$ only at 0 . We apply a result from Karlin (1959), Theorem B.3.5 on page 404, a delicate separation theorem, which says that there exists a strictly positive vector $e$ such that $\sum_{i} e_{i} y_{i} \leqslant 0$ for all $y$ in $Y$. By multiplying by a positive constant we can assume $\sum_{i} e_{i}=1$ and so $e \in \Delta$. The inner product condition says $a_{e x} \leqslant 0$ for all $x$ in $\mathbb{R}_{+}^{n}$ and so $a_{e j} \leqslant 0$ for all $j$. Thus, $a_{j e}=-a_{e j} \geqslant 0$ for all $j$. If any inequality were strict then $e$ would lie in $E_{+}$which is empty. So $e \in \Delta$ and $a_{j e}=0$ for all $j$, i.e. $e \in E_{0}$.

Finally, replacing the matrix $a_{i j}$ by its negative $-a_{i j}$ leaves the set $E_{0}$ unchanged and interchanges $E_{+}$and $E_{-}$. So the results for $E_{-}$follow from the above results for $E_{+}$.

Q.E.D.

In applying the functions of Lemma 1 to the cases of Theorem 2 we will repeatedly use versions of

3. Lemma. (a) Let $g(t)$ be a twice differentiable real function with $g^{\prime \prime}(t)$ uniformly bounded. If $\lim _{t \rightarrow \infty} g(t)$ exists and is finite then $\lim _{t \rightarrow \infty} g^{\prime}(t)=0$.

(b) Let $g_{n}$ be a sequence of real numbers. If $\lim _{n \rightarrow \infty} g_{n}$ exists and is finite then $\lim _{n \rightarrow \infty} \delta g_{n}\left(=g_{n+1}-g_{n}\right)=0$.

Proof: (b) is clear because if $\lim g_{n}=L$ then $\lim g_{n+1}-g_{n}=L-L=0$.

For (a), assume that $g^{\prime \prime}(t)$ is bounded by $M \geqslant 1$. If the $\lim _{t \rightarrow \infty} g^{\prime}(t)=0$ is not true, then we can choose a sequence $\left\{t_{n}\right\}$ approaching $\infty$ such that $g^{\prime}\left(t_{n}\right)$ is bounded away from zero. We can suppose $t_{n+1}>t_{n}+1$ and $g^{\prime}\left(t_{n}\right) \geqslant 2 \varepsilon$ with $0<\varepsilon<1$. Let $g_{2 n} \equiv g\left(t_{n}\right)$ and $g_{2 n+1}=g\left(t_{n}+\varepsilon / M\right)$. Because $g$ " is bounded by $M$, $g^{\prime}(t) \geqslant \varepsilon$ for $t_{n} \leqslant t \leqslant t_{n}+\varepsilon / M$. So by the mean value theorem $g_{2 n+1}-g_{2 n} \geqslant \varepsilon^{2} / M>$ 0 . By (b) $\lim _{n \rightarrow \infty} g_{n}$ does not exist and a fortiori $\lim _{t \rightarrow \infty} g(t)$ does not. Q.E.D.

We now turn to the main results of this section, the description of the fate of interior points in the two cases.

\footnotetext{
1 Parts of this argument are due to J. Hof bauer.
} 
4. Theorem. (Interior equilibrium case: $E_{0} \neq \emptyset$ )

(a) Assume the continuous dynamic (1.1). If $e \in E_{0}$ and $p \in \not{\Delta}$, then the function $I^{e}\left(\Phi^{t}(p)\right)$ remains constant as $t$ varies in $\mathbb{R}$. If $p \in \dot{\Delta}-E_{0}$, i.e. an interior nonequilibrium point, then the closure of the solution path is a compact invariant set containing $\omega_{\Phi}(p)$ and $\alpha_{\Phi}(p)$ and contained in $\Delta-E_{0}$. In particular, there is no equilibrium in this orbit closure.

(b) Assume the discrete dynamic (1.3). If $e \in E_{0}$ and $p \in \dot{\Delta}-E_{0}$ then $I^{e}\left(F^{t}(p)\right)$ is a strictly increasing function of $t$ in $\mathbb{Z} . F^{t}(p)$ approaches the boundary and $E_{0}$ as $t \rightarrow+\infty$ and $-\infty$, respectively, i.e. $\omega_{F}(p) \subset \partial \Delta$ and $\alpha_{F}(p) \subset E_{0}$.

Proof: If $e \in E_{0}$ then $e \in \mathbb{R}_{+}^{n}$ and $a_{e j}=-a_{j e}=0$ for all $j$. The function $I^{e}$ differs by a constant from $-L^{e}$. So by Lemma $1, d I^{e} / d t=0$ (using (1.1)) and $\delta I^{e} \geqslant 0$ (using (1.3)) at every interior point $p$. Furthermore, $\delta I^{e}>0$ at $p$ unless $a_{i p}=0$ for all $i$, i.e. $p \in E_{0}$. Because $F$ is invertible, $p \notin E_{0}$ implies $F^{t}(p) \notin E_{0}$ for all $t$ in $\mathbb{Z}$ and so $I^{e}\left(F^{t}(p)\right)$ is a strictly increasing function of $t$ unless $p \in E_{0}$.

For (a) assume that $p \in \stackrel{\Delta}{-} E_{0}$. Then for all $e \in E_{0}, I^{e}(p)$ is positive and finite. It is clear from the definition (1.8) that $I^{e}(p)$ approaches $\infty$ as $p$ approaches $\partial \Delta$. So the set $\left\{q \in \Delta: I^{e}(q)=I^{e}(p)\right.$ for all $\left.e \in E_{0}\right\}$ is a closed subset of $\Delta$, and so is compact, and lies entirely in $\dot{\Delta}-E_{0}$. Furthermore, it is an invariant set because the functions $I^{e}$ are invariant. As this set contains $p$ it contains the closure of the solution path through $p$.

For (b) assume that $p \in \ddot{\Delta}-E_{0}$. For all $e \in E_{0}$ the sequence $I^{e}\left(F^{t}(p)\right)$ is strictly

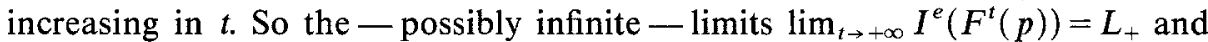
$\lim _{t \rightarrow-\infty} I^{e}\left(F^{t}(p)\right)=L_{-}$exist. Because $I^{e}$ is nonnegative and vanishes only at $e$, it is clear that $0 \leqslant L_{-}<L_{+} \leqslant \infty$. If $q \in \alpha_{F}(p)$, choose $\left\{t_{n}\right\}$ a decreasing sequence in $\mathbb{Z}$ with $\lim t_{n}=-\infty$ and $\lim F^{t_{n}}(p)=q . I^{e}(q)=\lim I^{e}\left(F^{t_{n}}(p)\right)=L_{-}<\infty$. Consequently, $q$ lies in the interior. Furthermore, applying Lemma 3 to $g_{n}=I^{e}\left(F^{t_{n}}(p)\right)$ we get that $\delta I^{e}(q)=\lim \delta I^{e}\left(F^{t_{n}}(p)\right)=0$. But on $\dot{\Delta}, \delta I^{e}$ vanishes only at points of $E_{0}$. Hence, $q \in E_{0}$ and so the compact invariant set $\alpha_{F}(p)$ is contained in $E_{0}$.

If $q \in \omega_{F}(p)$ and $\left\{t_{n}\right\}$ is an increasing sequence as above then we show $L_{+}=\infty$ and so $q$ lies in $\partial \Delta$. For if $L_{+}<\infty$ the above argument would again show $q \in E_{0}$. But for every $e$ in $E_{0} I^{e}(q)=L_{+}>0$. Thus, $\omega_{F}(p) \subset \partial \Delta$.

Remark: Result (b) with essentially this proof is due to Nagylaki.

5. Theorem. (No interior equilibrium case: $E_{0}=\emptyset$ ).

(a) Assume the continuous dynamic (1.1). If $e_{+} \in E_{+}, e_{-} \in E_{-}$and $p \in \Delta$ then $I^{e^{+}}\left(\Phi^{t}(p)\right)$ is strictly increasing and $I^{e_{-}}\left(\Phi^{t}(p)\right)$ is strictly decreasing in $t \in \mathbb{R} . \omega_{\Phi}(p)$ and $\alpha_{\Phi}(p)$ are subsets of $\partial \Delta$. In fact, if we define $J_{+}=\left\{i \in I: a_{i e}=0\right.$ for all $e$ in $\left.E_{+}\right\}$and $J_{-}=\left\{i \in I: a_{i e}=0\right.$ for all $e$ in $\left.E_{-}\right\}$, then $J_{+}$and $J_{-}$are proper subsets of $I$ and

$$
\begin{aligned}
& \omega_{\Phi}(p) \subset \Delta^{J_{-}} \\
& \alpha_{\Phi}(p) \subset \Delta^{J_{+}} .
\end{aligned}
$$

(b) Assume the discrete dynamic (1.3). If $e_{+} \in E_{+}$and $p \in \dot{\Delta}$ then $I^{e_{+}}\left(F^{t}(p)\right)$ is strictly increasing in $t \in \mathbb{Z} . \omega_{F}(p)$ and $\alpha_{F}(p)$ are subsets of $\partial \Delta$. For the latter we have

$$
\alpha_{F}(p) \subset \Delta^{J_{+}} .
$$


Proof: That $d I^{e^{+}} / d t>0, d I^{e^{-}} / d t<0$ and $\delta I^{e_{+}}>0$ at all interior points follow from Lemma 1.

To prove (1.10) we apply Lemma 3(a) noting that by the derivative calculation (1.9) the second derivative of $I^{e}+\left(\Phi^{t}(p)\right)$ is clearly bounded. Because $I^{e+\left(\Phi^{t}(p)\right)}$ is increasing in $t$ and nonnegative it has a finite limit $L_{+}$as $t$ approaches $-\infty$. So if $q \in \alpha_{\Phi}(p), I^{e_{+}}(q)=L_{+}$is finite and $d I^{e_{+}} / d t=0$ at $q$. This implies that $\operatorname{supp}(q) \supset \operatorname{supp}\left(e_{+}\right)$and $\alpha_{i e_{+}}=0$ for all $i$ in $\operatorname{supp}(q)$, i.e. $\operatorname{supp}(q) \subset J_{+}$. Hence, $\alpha_{\Phi}(p) \subset \Delta^{J_{+}}$. By definition of $E_{+}, J_{+}$is a proper subset of $I$ and so $\Delta^{J_{+}} \subset \partial \Delta$. The result for $\omega_{\Phi}$ follows by replacing $a_{i j}$ by $-a_{i j}$.

To prove (1.11) we apply Lemma 3(b) and in the same pattern as the above argument we get that for $q \in \alpha_{F}(p), I^{e_{+}}(q)$ is finite and $\delta I^{e_{+}}=0$ at $q$. This implies that $\operatorname{supp}\left(e_{+}\right) \subset \operatorname{supp}(q) \subset J_{+}$as in the continuous case. If $q \in \omega_{F}(p)$ then $\lim _{t \rightarrow+\infty} I^{e_{+}}\left(F^{t}(p)\right)<\infty$ implies $q \in \Delta^{J_{+}}$while $\lim _{t \rightarrow \infty} I^{e_{+}}\left(F^{t}(p)\right)=\infty$ implies that $\operatorname{supp}(q)$ cannot contain $\operatorname{supp}\left(e_{+}\right)$. So in either case $q_{i}=0$ for some $i$ and $q \in \partial \Delta$.

Remark: Because $I^{e^{-}-}$need not be monotone on solution paths in the discrete case, we cannot prove that $\omega_{F}$ is contained in $\Delta^{J_{-}}$. This is a question to which we will return. On the other hand, we get a sharper result than was stated for points of $\alpha_{F}(p)$. For $q \in \alpha_{F}(p)$ we saw that $\delta I^{e^{e}}$ is zero at $q$ and so:

$$
q \in \boldsymbol{\alpha}_{F}(p) \Rightarrow a_{i q}=0 \quad \text { for all } i \in \operatorname{supp}\left(e_{+}\right) .
$$

These results reveal in detail the difference between the two sorts of dynamics. In the interior equilibrium, continuous case $\dot{\Delta}$ is filled with invariant manifolds containing no equilibria. All interior equilibria are stable, though not asymptotically stable, because $I^{e}$ is an invariant function. We will examine this conservative behavior in Sect. 3. By contrast for the discrete time dynamics we have:

6. Corollary. Assume the discrete time dynamic. If $p$ is an interior nonequilibrium point then as $t \rightarrow \infty$ in $\mathbb{Z} F^{t}(p)$ approaches the boundary. Any closed invariant subset of $\Delta$ contains equilibria.

Proof: $\omega_{F}(p) \subset \partial \Delta$ for nonequilibrium interior $p$ in both cases. Now let $C$ be a closed invariant subset of $\Delta$ and choose $p \in C$ with minimal support, i.e. if $J=\operatorname{supp}(p)$ then there does not exist $q \in C$ with $\operatorname{supp}(q)$ a proper subset of $J$. It follows that $p$ is an equilibrium. Assume not. Then $p$ is an interior nonequilibrium point for the dynamic restricted to the strategies in $J$, and so $\omega_{F}(p) \subset \partial \Delta^{J}$. Because $C$ is closed and invariant, $p \in C$ implies $\omega_{F}(p) \subset C$. The points of $\omega_{F}(p)$ lie in $C$ and have support smaller than $J$. This contradicts the minimality of $p$.

Q.E.D.

Remark. It follows that the only periodic points, points $p$ such that $F^{t}(p)=p$ for some $t \in \mathbb{Z}-0$, are equilibria.

Now in the no-interior-equilibrium case, (1.10) implies that strategies $i \not J_{-}$, i.e. $a_{i e_{-}}<0$ for some $e_{-} \in E_{-}$, are eliminated by competition. In fact $p_{i}=0$ on $\Delta^{J_{-}}$ and $\omega_{\Phi}(p) \subset \Delta^{J}$ - imply that beginning at any interior point $p$,

$$
\lim _{i \rightarrow \infty} \Phi^{t}(p)_{i}=0 \quad \text { for all } i \notin J_{-}
$$


In particular, the queer recurrence behavior described in Akin and Hofbauer (1982) cannot occur for anti-symmetric models.

The question arises whether the same result is true for the discrete time dynamic. The answer appears to depend on the size of the entries $a_{i j}$.

Consider the payoff matrix parametrized by $1>\tau>0$ :

$$
\tau\left(\begin{array}{rrrr}
0 & --\frac{1}{2} & \frac{1}{2} & -\frac{1}{4} \\
\frac{1}{2} & 0 & 1 & -1 \\
-\frac{1}{2} & -1 & 0 & 1 \\
\frac{1}{4} & 1 & -1 & 0
\end{array}\right) .
$$

To the paper-rock-scissors game we have added a new strategy (labelled 0) which behaves somewhat like paper (strategy 1). It is easy to check that:

$$
\begin{aligned}
& E_{-}=\left\{\left(0, \frac{1}{3}, \frac{1}{3}, \frac{1}{3}\right)\right\} \\
& E_{+}=\left\{\left(\frac{4}{7}, 0, \frac{1}{7}, \frac{2}{7}\right)\right\} .
\end{aligned}
$$

We will see below that if $\tau>0$ is sufficiently small then $\lim _{n \rightarrow \infty} F_{\tau}^{n}(p)_{0}=0$ for all interior points $p$. In other words, the analogue of (1.13) holds and the new strategy is eliminated. However, for $\tau$ near 1 , e.g. $\tau=0.9$, numerical results suggest most interior orbits do not eliminate 0 in the limit. Instead of approaching the "edge cycle" $3 \rightarrow 2 \rightarrow 1 \rightarrow 3$ of the original game, most strategies appear to approach the "edge-cycle" $3 \rightarrow 2 \rightarrow 0 \rightarrow 1 \rightarrow 3$.

In Sect. 2, we will discuss the general recurrence patterns for the discrete time model in the nondegenerate case. We conclude this section by introducing the nondegenerate case and proving the analogue of (1.13) for $\tau$ small enough.

Recall that $\operatorname{det}\left(a_{i j}\right)=\operatorname{det}\left(a_{j i}\right)=\operatorname{det}\left(-a_{i j}\right)=(-1)^{n} \operatorname{det}\left(a_{i j}\right)$, where det stands for the determinant. So if $n$ is odd the determinant of the antisymmetric matrix $a_{i j}$ is zero. If $n$ is even then by perturbing the entries slightly we can preserve antisymmetry and get a nonzero determinant. Hence, the set of nondegenerate antisymmetric matrices is open and dense in the set of all antisymmetric matrices when we define $\left(a_{i j}\right)$ to be a nondegenerate antisymmetric matrix when for all subsets $J$ of $I=\{1, \ldots, n\}$ containing an even number of elements:

$$
\operatorname{det}\left(a_{i j}: i, j \in J\right) \neq 0 .
$$

In other words, the even dimensional principal minors of $a_{i j}$ are all nonzero.

7. Lemma. Assume $a_{i j}$ is a nondegenerate antisymmetric matrix and $J \subset I$. Suppose there exists a nonzero vector $x$ with $\operatorname{supp}(x) \subset J$ and $a_{i x}=0$ for all $i$ in $J$. Then $J$ has an odd number of elements, $a_{i x} \neq 0$ for $i \notin J$ and $x_{i} \neq 0$ for $i \in J$ (i.e. $\operatorname{supp}(x)=J$ ). Furthermore, if $J$ has an odd number of elements then such a vector exists and is unique up to a nonzero scalar multiple.

Proof: If $J$ is even then the homogeneous system of equations $\sum_{j \in J} a_{i j} x_{j}=0(i \in J)$ has only the trivial solution by (1.14). If $J$ contains $2 m+1$ elements then $\left(a_{i j}: i, j \in\right.$ $J$ ) has rank $2 m$ by (1.14) and so the system has a one dimensional solution space. If $\left\{x_{j}: j \in J\right\}$ is a nonzero solution then defining $x_{i}=0$ for $i \notin J$ yields a nonzero vector $x$ in $\mathbb{R}^{n}$ with $\operatorname{supp}(x) \subset J$ and $a_{i x}=0$ for $i \in J$. If $x_{j}=0$ for some $j \in J$ then 
we would have a solution for $J-\{j\}$ which is even. If $a_{i x}=0$ for some $i \notin J$ then we would have a solution for $J \cup\{i\}$ which is even. As the even cases admit only the trivial solution it must be that $\operatorname{supp}(x)=J$ and $a_{i x} \neq 0$ for $i \notin J$. Q.E.D.

8. Proposition. Assume $a_{i j}$ is a nondegenerate antisymmetric matrix.

(a) If $e$ is an equilibrium, i.e. $e \in \Delta, a_{i e}=0$ for all $i \in \operatorname{supp}(e)=J$, then $J$ has an odd number of elements and $e^{\prime}$ is the only equilibrium with support equal to $J$. Furthermore, $a_{i e} \neq 0$ for $i \notin J$. In particular, there are only finitely many equilibria.

(b) If $J \subset I$ there is a unique vector $e_{+} \in \Delta$ with $J_{+} \equiv \operatorname{supp}\left(e_{+}\right) \subset J$ and $a_{i e_{+}} \geqslant 0$ for all $i$ in $J$ and there is a unique vector $e_{-} \in \Delta$ with $J_{-} \equiv \operatorname{supp}\left(e_{-}\right) \subset J$ and $a_{i e_{-}} \leqslant 0$ for all $i$ in $J$. Either $e_{+}=e_{-}$, with $J_{+}=J=J$ and $a_{i e_{+}}=a_{i e_{-}}=0$ for all $i$ in $J$ ( $\Delta^{J}$ has an interior equilibrium) or $J_{+}$and $J_{-}$are proper subsets of $J$ with $a_{i e_{+}}>0$ for all $i \in J-J_{+}$and $a_{i e_{-}}<0$ for all $i \in J-J_{-}$( $\Delta^{J}$ has no interior equilibrium).

(c) Assume the continuous dynamic (1.1) and let $p \in \Delta$ with $\operatorname{supp}(p)=J$. $\omega_{\Phi}(p) \subset \dot{\Delta}^{J_{-}}$and $\alpha_{\Phi}(p) \subset \dot{\Delta}^{J_{+}}$.

(d) Assume the discrete dynamic (1.3) and let $p \in \Delta$ with $\operatorname{supp}(p)=J . \alpha_{F}(p)=$ $\left\{e_{+}\right\}$and either $p=e_{+}=e_{-}$or $\omega_{F}(p) \subset\left\{x \in \Delta^{J}: x_{i}=0\right.$ for some $\left.i \in J_{+}\right\}$.

Proof: By Lemma 7, $a_{i x}=0$ for $i \in J$ and $\operatorname{supp}(x)=J$ has no solution if $J$ is even and a one dimensional manifold of solutions if $J$ is an odd subset. In the latter case there is an equilibrium with support $J$ if and only if the line of solutions intersects $\mathbb{R}_{+}^{n}-0$. If so, normalizing to $\sum_{i} x_{i}=1$, i.e. $x \in \Delta$, yields a unique solution $x=e$. In particular, there is at most one equilibrium for any support set and so only finitely many equilibria.

In proving (b), (c) and (d) we can assume $J=I$, as the general result is obtained by restricting to the subsystem with strategies only in $J$.

Since $E_{0}, E_{+}$and $E_{-}$are convex sets of equilibria and since there are only finitely many equilibria, each set is either empty or contains exactly one point. In the interior equilibrium case where $E_{0} \neq \emptyset$ then $e_{+}=e_{-}$is the single point in $E_{0}$. If $p$ is an equilibrium on the boundary then $a_{i p} \neq 0$ for $i \notin \operatorname{supp}(p)$ and so the conditions $e \in \Delta$ and $a_{i e}=0$ for all $i$ define this interior equilibrium uniquely. When $E_{0}=\varnothing$, then $E_{+}$and $E_{-}$each contain a single point labelled $e_{+}$and $e_{-}$ respectively.

For (c), in the interior equilibrium case with $J=I \Delta^{J_{+}}=\dot{\Delta}^{J_{-}}=\dot{\Delta}$ and the result follows from Theorem 4(a). When there is no interior equilibrium, Theorem 5(a) implies $\omega_{\Phi}(p) \subset \Delta^{J_{-}}$and $\alpha_{\Phi}(p) \subset \Delta^{J_{+}}$. Furthermore the proof shows that $I^{e_{-}}$and $I^{e_{+}}$are finite on $\omega_{\Phi}(p)$ and $\alpha_{\Phi}(p)$ respectively. So the sets lie in the interiors.

For (d), in the interior case with $J=I$, the result follows from Theorem 4(b). When there is no interior equilibrium Theorem 5(b) implies that $\omega_{F}(p)$ is in the boundary and $\alpha_{F}(p) \subset \Delta^{J_{+}}$. Furthermore, by (1.12) if $q \in \alpha_{F}(p), a_{i q}=0$ for all $i \in J_{+}$so $q$ is the equilibrium with support $J_{+}$, i.e. $q=e_{+}$.

Finally, if the increasing sequence $I^{e^{+}}\left(F^{t}(p)\right)$ had a finite limit then the same argument would show $\omega_{F}(p)=\left\{e_{+}\right\}$. But for $q \in \omega_{F}(p), I^{e_{+}}(q)>I^{e_{+}}(p)>0$ because $p$ is in the interior. Consequently, $\lim _{t \rightarrow \infty} I^{e^{+}}\left(F^{t}(p)\right)=\infty$ and so $\omega_{F}(p) \cap$ $\left\{x \in \Delta^{J}: x_{i}=0\right.$ for $\left.i \in J_{+}\right\}=\varnothing$.

Q.E.D.

In preparation for our remaining result we require another separation argument. 
9. Lemma. Assume $a_{i j}$ is a nondegenerate matrix with no interior equilibriuri. Assume $a_{k e_{-}}<0$, i.e. $k \notin \operatorname{supp}\left(e_{-}\right)$. There exists a vector $b \in \mathbb{R}^{n}$ such that $a_{b j}>0$ for all $j$, $b_{i}>0$ for all $i \neq k$ and $b_{k}<0$.

Proof: Let $D$ be the $n \times n$ diagonal matrix with $D_{i i}=1$ for $i \neq k$ and $D_{k k}=-1$. Define the $n \times 2 n$ matrix $B$ by the block form

$$
B=(A, D) \text {. }
$$

We are looking for a vector $b \in \mathbb{R}^{n}$ such that $b B$ is a positive vector. By Gale (1960), Theorem 2.9 page 48, if such a vector $b$ does not exist then there exist vectors $x, y \in \mathbb{R}_{+}^{n}$ not both zero such that $A x+D y=0$, i.e. $a_{i x}=-y_{i} \leqslant 0$ for $i \neq k$ and $a_{k x}=y_{k} \geqslant 0$. Clearly, $x=0$ implies $y=0$, too, and so $x \neq 0$. We can multiply by a positive constant to get $x \in \Delta$ such that $a_{i x} \leqslant 0$ for $i \neq k$ and $a_{k x} \geqslant 0$. Because $e_{-} \in \mathbb{R}_{+}^{n}$ and $k \notin \operatorname{supp}\left(e_{-}\right)$it follows that $a_{x e_{-}}=-a_{e_{-} x} \geqslant 0$. But $a_{i e_{-}} \leqslant 0$ for all $i$ and so $a_{x e_{-}} \leqslant 0$ with strict inequality unless $\operatorname{supp}(x) \subset \operatorname{supp}\left(e_{-}\right)$. Now with $J=$ $\operatorname{supp}\left(e_{-}\right), e_{-}$is the unique vector $x$ in $\Delta$ with $\operatorname{supp}(x) \subset J$ and $a_{i x} \leqslant 0$ for all $i$ in $J$ by Theorem $8(\mathrm{~b})$. So $x=e_{-}$. This is impossible because $a_{k x} \geqslant 0$ while $a_{k e_{-}}<0$. Consequently, the vector $b$ exists.

Q.E.D.

10. Theorem. Assume $a_{i j}$ is a nondegenerate symmetric matrix (satisfying (1.2)). For any $0<\tau \leqslant 1$ let $F_{\tau}$ be the map obtained from (1.4) by replacing $a_{i p}$ by $\tau a_{i p}$. Consider the discrete dynamical system obtained from (1.3) by this replacement. There exists $\tau_{0}>0$ such that if $0<\tau \leqslant \tau_{0}$ then $\operatorname{supp}(p)=J$ implies $\omega_{F_{\tau}}(p) \subset \Delta^{J}$.

Proof: It is sufficient to find a $\tau_{0}>0$ which will work when $p$ is interior. Then we apply the result to each support subset and use the minimum $\tau_{0}$ from among those so found. In the interior equilibrium case $J_{-}$is all of $I$ and the result is trivial (use $\tau_{0}=1$ ). Finally, in the no interior equilibrium case look at $k \notin \operatorname{supp}\left(e_{-}\right)$ i.e. $a_{k e_{-}}<0$. We will find a $\tau_{k}>0$ such that when $\tau \leqslant \tau_{k}$, and $p \in \dot{\Delta}, q \in \omega_{F_{\tau}}(p)$ implies $q_{k}=0$. The result then follows by choosing $\tau_{0}=$ minimum $\tau_{k}$ as $k$ varies over $I-\operatorname{supp}\left(e_{-}\right)$.

With $k$ fixed we choose the vector $b$ given by Lemma 9 and try to apply

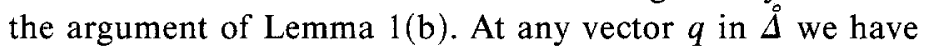

$$
\delta L^{b}(q)=\sum b_{i} \ln \left(1+\tau a_{i q}\right) .
$$

But now the concavity of the $\log$ is no help. Instead apply Taylor's theorem to write $\ln (1+\tau u)=\tau u+\tau^{2} R(\tau, u)$ where $|R(\tau, u)| \leqslant 1$ for $|u| \leqslant 1$ and $\tau \leqslant 1 / 2$.

Define

$$
\tau_{k}=\frac{\min \left\{a_{b j}\right\}}{2\left(\sum\left|b_{j}\right|\right)} .
$$

Then for all $q$ in $\Delta \tau_{k} \leqslant a_{b q} / 2\left(\sum\left|b_{j}\right|\right)$ and so for $0<\tau \leqslant \tau_{k}$

$$
\begin{aligned}
\delta L^{b} & =\tau\left(a_{b q}+\tau \sum b_{j} R\left(\tau, a_{j q}\right)\right) \geqslant \tau\left(a_{b q}-\frac{1}{2} a_{b q}\right) \\
& =\tau a_{b q} / 2>0 .
\end{aligned}
$$

Consequently, if $\tau \leqslant \tau_{k}, L^{b}\left(F_{\tau}^{t}(p)\right)$ is increasing in $t$. If $q \in \partial \Delta$ and $q_{k}>0$ then as $x$ nears $q$ the second term of

$$
L^{b}(x)=\sum_{j \neq k} b_{j} \ln x_{j}+b_{k} \ln x_{k}
$$


remains finite while the first approaches $-\infty$ because $b_{j}>0$ for all $j \neq k$. As $L^{b}\left(F_{\tau}^{t}(p)\right)$ is finite and increasing in $t$, no subsequence of $F_{\tau}^{t}(p)$ can approach $q$ as $t \rightarrow \infty$.

So $q_{k}=0$ at every point of $\omega_{F_{\tau}}(p)$.

Q.E.D.

Remark: In particular, for nondegenerate $a_{i j}$ and $\tau>0$ sufficiently small the analogue of (1.13) holds: Beginning at any interior point:

$$
\lim _{t \rightarrow \infty} F_{\tau}^{t}(p)_{i}=0 \quad \text { for all } i \notin J_{-} .
$$

\section{Attractors in the discrete system}

Let us begin by describing some concepts from topological dynamics due to Conley and Smale.

If $F$ is a homeomorphism of a compact metric space $X$ onto itself we define an $\varepsilon$-chain to be a sequence $\left\{x_{0}, \ldots, x_{N}\right\}$ in $X$ such that $d\left(x_{i}, F\left(x_{i-1}\right)\right)<\varepsilon$ for $i=1, \ldots, N$, where $d$ is the metric on $X$. Define the quasiordering on points of $X$ :

$$
\begin{aligned}
& x \gg y \quad \text { if for every } \varepsilon>0 \text { there exists an } \\
& \varepsilon \text {-chain }\left\{x_{1}, \ldots, x_{N}\right\} \text { with } x=x_{1} \text { and } y=x_{N} .
\end{aligned}
$$

The ordering is clearly transitive and it is reflexive because $\{x\}$ is an $\varepsilon$-chain connecting $x$ with itself. However, the ordering is usually not antisymmetric. Instead, the associated equivalence relation is defined by:

$$
x \approx y \quad \text { if } x \gg y \text { and } y \gg x
$$

and $\gg$ induces a partial ordering on equivalence classes.

By uniform continuity $x \gg y$ implies $F(x) \gg F(y)$ and $F^{-1}(x) \gg F^{-1}(y)$. Clearly, the implications hold with $\gg$ replaced by $\approx$ as well.

Obviously, $x \gg F(x)$ and more generally $x \gg F^{n}(x)$ when $n>0$. A point $x$ is called chain recurrent if $F(x) \gg x$ and so $x \approx F(x)$. An invariant set $A$, i.e. a set with $F(A)=A$, is called a chain recurrent set if $x_{1}, x_{2} \in A$ implies $x_{1} \approx x_{2}$. For example, if $p \in X$ and we define the limit points sets $\alpha(p)$ and $\omega(p)$ by (1.5) and (1.6) then $\alpha(p)$ and $\omega(p)$ are chain recurrent sets. For if $x_{1}, x_{2} \in \omega(p)$ for example, and $\varepsilon>0$, choose $\delta>0$ so that $d\left(x_{1}, y\right)<\delta$ implies $d\left(F\left(x_{1}\right), F(y)\right)<\varepsilon$. There exists $n_{1}>0$ such that $d\left(x_{1}, F^{n_{1}}(p)\right)<\delta$ and $n_{2}>n_{1}+1$ such that $d\left(x_{2}, F^{n_{2}}(p)\right)<\varepsilon$. Then $\left\{x_{1}, F^{n_{1}+1}(p), F^{n_{1}+2}(p), \ldots, F^{n_{2}-1}(p), x_{2}\right\}$ is an $\varepsilon$-chain from $x_{1}$ to $x_{2}$.

Notice that a chain recurrent set consists entirely of chain recurrent points because $x \in A$ implies $F(x) \in A$. Also, it is easy to check that the closure of a chain recurrent set is chain recurrent. If $x$ is a chain recurrent point then its $\approx$ equivalence class is invariant because $x \approx y$ implies $F^{-1}(y) \approx F^{-1}(x) \approx x \approx F(x) \approx$ $F(y)$. So this class is a chain recurrent set. It is clearly the largest chain recurrent set containing $x$ and so is closed. The equivalence classes of chain recurrent points are called the basic sets of $F$. 
The other key notion is defined in:

1. Lemma (Smale): Define $\alpha(F)$ to be the closure in $X$ of $\bigcup\{\alpha(p): p \in X\}$.

For any closed invariant set $A$ in $X$ the following three conditions are equivalent:

(1) There exists a closed subset $Q$ of $X$ such that $F(Q) \subset$ Interior $_{X} Q$ and $A=\bigcap_{n \geqslant 0} F^{n}(Q)$.

(2) $A \cap \alpha(F)$ is relatively open in $\alpha(F)$ and $p \notin A$ implies $\alpha(p) \cap A=\varnothing$.

(3) There exists a closed subset $Q$ of $X$ with $A \subset$ Interior $_{X} Q$ and $A=$ $\bigcap_{n \geqslant 0} F^{n}(Q)$.

If $A$ satisfies these conditions then it is called an attractor. A repellor is defined to be an attractor for the map $F^{-1}$.

Proof: $(1) \Rightarrow(2)$ : Given $Q$ as in (1), $F^{n}(Q)$ is a decreasing sequence of sets with intersection $A$. So if $p \notin A$ then $p \notin F^{n}(Q)$ for $n$ sufficiently large, i.e. $F^{-n}(p) \notin Q$ for $n$ large and so $\alpha(p)$ is disjoint from Interior $Q$ and in particular from A. Also, $A \cap \alpha(F)=$ Interior $Q \cap \alpha(F)$ and so $A \cap \alpha(F)$ is relatively open in $\alpha(F)$.

$(2) \Rightarrow(3)$ : Because $A \cap \alpha(F)$ is closed and is relatively open we can choose $Q$ a closed set containing $A$ in its interior and such that $Q \cap \alpha(F)=A \cap \alpha(F)$, e.g. $\alpha(F)-(A \cap \alpha(F))$ is closed in $X$ and disjoint from $A$ and so its complement contains a closed neighborhood of $A . A \subset \bigcap_{n \geqslant 0} F^{n}(Q)$ because $A$ is invariant. On the other hand, if $p \in \bigcap_{n \geqslant 0} F^{n}(Q)$ then $F^{-n}(p) \in Q$ for all $n \geqslant 0$. So $\alpha(p) \subset$ $Q \cap \alpha(F)=A \cap \alpha(F)$. By (2) $\alpha(p) \subset A$ implies $p \in A$.

$(3) \Rightarrow(1)$ : See Smale (1970) Lemma 4.2 page 292.

Q.E.D.

Remark: If $Q$ satisfies condition (1) for $A$, let $\tilde{Q}=X$-Interior $Q$. Clearly $F(Q) \subset \operatorname{Int} Q \Rightarrow Q \subset F^{-1}(\operatorname{Int} Q) \Rightarrow F^{-1}(\tilde{Q}) \subset X-Q \subset$ Int $\tilde{Q}$. Then $B=$ $\bigcap_{n \geqslant 0} F^{-n}(\tilde{Q})$ is a closed invariant set called the repellor dual to the attractor $A$. It is easy to check that if $p \in X-(A \cup B)$ then $\alpha(p) \subset B$ and $\omega(p) \subset A$.

The relation between chain recurrence and attractors is:

2. Lemma. If $A$ is an attractor, $x \in A$ and $x \gg y$ then $y \in A$.

Proof: If $Q$ satisfies condition (1) for $A$ and $\varepsilon>0$ is smaller than the distance between the disjoint compacta $F(Q)$ and $X$ - Int $Q$ then any $\varepsilon$ chain starting in $Q$ remains in $Q$. For every $n \geqslant 0$ apply this argument to $F^{n}(Q)$ to show $x \gg y$ and $x \in A$ implies $y \in F^{n}(Q)$ for all $n$. Hence, $y \in A$.

Q.E.D.

In addition to their tremendous theoretical utility, the ideas associated with chain recurrence are very suggestive for applications. The flow-with-errors, as Conley calls it, is likely to be a more believable picture of the world we are trying to model than the precise flow itself. Thus, the chain recurrence structure is likely to be the most reliable information that the model imparts. The major reference for these ideas is Conley's (1978) beautiful lecture notes.

In applying these ideas to the map $F: \Delta \rightarrow \Delta$ of (1.4) with $a_{i j}$ a nondegenerate antisymmetric matrix, we will be able to capture all of the chain recurrence structures by using the equilibria. This is because by Proposition $1.8(\mathrm{~d}) \alpha(p)$ is an equilibrium for every $p$ in $\Delta$. Notice that the behavior of the forward and backward dynamics of $F$ are completely different. This contrasts with the flow $\Phi^{t}$ associated with $a_{i j}$ because $\Phi^{-t}$ is just the flow for $-a_{i j}$. 
For the rest of the section we will assume:

$a_{i j}$ is a nondegenerate, antisymmetric matrix.

We begin with some notation. If $e$ is an equilibrium for $a_{i j}$ then define:

$$
\begin{aligned}
I_{+}(e) & =\left\{i: a_{i e}>0\right\} \\
I_{-}(e) & =\left\{i: a_{i e}<0\right\} \\
I_{0}(e) & =\operatorname{supp}(e) \\
I_{0+}(e)=I_{0} \cup I_{+} & \text {and } I_{0-}=I_{0} \cup I_{-} .
\end{aligned}
$$

The following is just a restatement of Proposition 1.8(a) and (b) using this notation.

3. Proposition. (a) For every equilibrium e, the set $I=\{1, \ldots, n\}$ is partitioned into the mutually disjoint sets $I_{0}(e), I_{+}(e)$ and $I_{-}(e)$.

(b) If $J \subset I$ there are unique equilibria $e_{+}$and $e_{-}$associated with $J$ such that

$$
\begin{aligned}
& I_{0}\left(e_{+}\right) \subset J \subset I_{0+}\left(e_{+}\right) \\
& I_{0}\left(e_{-}\right) \subset J \subset I_{0-}\left(e_{-}\right) .
\end{aligned}
$$

Furthermore, either $e_{+}=e_{-}$and $J=I_{0}\left(e_{+}\right)=I_{0}\left(e_{-}\right)$or $I_{0}\left(e_{+}\right)$and $I_{0}\left(e_{-}\right)$are proper subsets of $J$.

Now we define a relation between equilibria. If $e_{1}$ and $e_{2}$ are equilibria for $a_{i j}$ then

$$
e_{1}>e_{2} \quad \text { if } I_{0}\left(e_{2}\right) \subset I_{0+}\left(e_{1}\right) .
$$

In other words, $e_{1}>e_{2}$ if $a_{i e_{1}} \geqslant 0$ for all $i$ in the support of $e_{2} .>$ is reflexive but is usually not transitive.

4. Theorem. (a) For equilibria $e_{1}, e_{2}$ in $\Delta, e_{1} \gg e_{2}$ if and only if there is a sequence $p_{1}, p_{2}, \ldots, p_{N}$ of equilibria with $p_{1}=e_{1}, p_{N}=e_{2}$ and $p_{1}>p_{2}>\cdots>p_{N}$.

(b) Call $E$ a terminal set of equilibria if $e_{1} \in E$ and $e_{1}>e_{2}$ implies $e_{2} \in E$. When $E$ is terminal define the closed subcomplex of $\Delta, A(E)$ by

$$
A(E)=\bigcup\left\{\Delta^{I_{0+}(e)}: e \in E\right\} .
$$

$A(E)$ is an attractor for $F$ and $E$ is the set of equilibria contained in $A(E)$.

(c) An equilibrium $e$ is called minimal if $e \gg e_{1}$ implies $e \approx e_{1}$. The set $E_{M}$ of minimal equilibria is terminal and $e_{1}, e_{2} \in E_{M}$ imply $e_{1} \approx e_{2} . A\left(E_{M}\right)$ is the unique chain recurrent attractor for $F . p \gg x$ for all $p$ in $\Delta$ and all $x$ in $A\left(E_{M}\right)$. So $A\left(E_{M}\right)$ is contained in every attractor.

(d) If $A$ is an attractor for $F$ then $A=A(E)$ for the terminal set $E=\{e \in A$ : $e$ is an equilibrium\}.

Proof: If $e_{1}>e_{2}$ then let $J=I_{0+}\left(e_{1}\right)$. For $J e_{+}$is $e_{1}$ and so for any point $p$ with $\operatorname{supp}(p)=J, \alpha_{F}(p)=e_{1}$. Given $\varepsilon>0$ we can choose $p$ with $\operatorname{supp}(p)=J$ and $\left|p-e_{2}\right|<\varepsilon$ because $e_{2}$ lies in $\Delta^{J}$. Choose $n>0$ such that $\left|F^{-n}(p)-e_{1}\right|<\varepsilon$. Then $\left\{e_{1}, F^{-n}(p), F^{-n+1}(p), \ldots, F^{-1}(p), e_{2}\right\}$ is an $\varepsilon$-chain from $e_{1}$ to $e_{2}$. So $e_{1} \gg e_{2}$. 
Now we turn to (b). As $A(E)$ is a union of faces $\Delta^{J}$ it is closed and invariant. $\alpha(F)$ is the finite set of all equilibria and so $A(E) \cap \alpha(F)$ is relatively open in $\alpha(F)$ (every subset is open and closed in $\alpha(F)$ ). If $e_{1}$ is an equilibrium in $A(E)$ then $e_{1} \in \Delta^{I_{0+}(e)}$ for some $e$ in $E$. So $e>e_{1}$ and $e_{1} \in E$ because $E$ is terminal. Finally, if $\operatorname{supp}(p)=J$ then $\alpha(p)=e_{+}$for $J$. So $\alpha(p) \cap A(E) \neq \varnothing$, i.e. $e_{+} \in A(E)$, implies $e_{+} \in E$ and so $p \in \Delta^{I_{0+}\left(e_{+}\right)} \subset A(E)$. Thus, $A(E)$ satisfies condition (2) of Smale's lemma.

Now we complete the proof of (a). If $e_{1}$ is an equilibrium define $E$ to be the set of equilibria $e_{2}$, such that there is a sequence $p_{1}, \ldots, p_{N}$ of equilibria with $e_{1}=p_{1}, e_{2}=p_{N}$ and $p_{1}>p_{2} \cdots>p_{N}$. $E$ is clearly terminal and so by (b) $A(E)$ is an attractor. If $e$ is equilibrium and $e_{1} \gg e$ then $e \in A(E)$ by Lemma 2. So $e \in E$ by (b). This completes the proof of (a).

(c) Suppose $e$ is minimal and $e>e_{1}$. We show that $e_{1}$ is minimal. Assume $e_{1} \gg e_{2}$. Then by (a), $e \gg e_{1}$ and $e \gg e_{2}$. So $e_{1} \approx e \approx e_{2}$. Thus, $e_{1}$ is minimal and $E_{M}$ is terminal. The hard part is to show that all the equilibria in $E_{M}$ are equivalent. The key is to consider the vertices of the simplex. Letting $i$ stand for the vertex whose $i$ coordinate is one, we recall that $i$ is an equilibrium. Furthermore, if $i \neq j$ then because $J=\{i, j\}$ is an even set $\Delta^{J}$ has no interior equilibrium and so $e_{+} \neq e_{-}$ for $J$. Thus $e_{+}=i$ and $e_{-}=j$ (or the other way) and so $i>j$. In other words, any two vertices are comparable with respect to $>$. Also if $e$ is an equilibrium and $i \in I_{0+}(e)$ then $e>i$. Now let $e_{1}, e_{2} \in E_{M}$. Choose vertices such that $e_{1}>i$ and $e_{2}>j$. Because $i$ and $j$ are comparable we can suppose $i>j$. Hence, $e_{1} \gg j$ and $e_{2} \gg j$. Because $e_{1}, e_{2} \in E_{M}$ we have $e_{1} \approx j \approx e_{2}$.

$A\left(E_{M}\right)$ is an attractor by (b). If $p \in A\left(E_{M}\right)$ and $\operatorname{supp}(p)=J$ then $\alpha(p)=e_{+}$ for $J$ and $\omega(p)$ is a closed invariant subset of $\Delta^{J}$ and so contains some equilibrium $e$ of $\Delta^{J}$. Because $e_{+} \in \alpha(p)$ and $e \in \omega(p), e_{+} \gg p \gg e$. Because $A\left(E_{M}\right)$ is invariant $e_{+} \in A\left(E_{M}\right)$ and so is in $E_{M}$. Hence, $e \approx e_{+}$and $p$ is equivalent to both. So all the points of $A\left(E_{M}\right)$ are chain equivalent to some equilibrium of $E_{M}$ and all the equilibria of $E_{M}$ are equivalent to each other. Thus, $A\left(E_{M}\right)$ is chain recurrent.

If $p \in \Delta$ then $\omega(p)$ contains some equilibrium $p_{1}$ and $p \gg p_{1}$. If $p_{1}$ is not minimal then there exists an equilibrium $p_{2}$ with $p_{1} \gg p_{2}$ and not $p_{1} \approx p_{2}$. We continue inductively building a sequence $p \gg p_{1} \gg p_{2} \cdots \gg p_{N}$ with $p_{i} \neq p_{i+1}$ for $i=1, \ldots, N-1$. Since there are only finitely many equilibria and this process cannot cycle by transitivity of $\gg$ it must terminate at a minimal equilibrium. So $p \gg p_{N}$ with $p_{N} \in A\left(E_{M}\right)$.

Finally, if $A$ is an attractor and $E$ is the set of equilibria in $A$ then $E$ is terminal by Lemma 2 and so $A$ and $A(E)$ contain the same set $E$ of equilibria. If $p$ lies in either set then $e=\alpha(p)$ is in $E$ and $e \gg p$. So $p$ lies in both sets by Lemma 2 again.

Q.E.D.

The utility of this result comes from the simplicity of the relation (2.3) on the finite set of equilibria. Using $>$ to generate a transitive relation in part (a) we obtain the chain ordering $\gg$ on equilibria. Terminality of a set $E$ is defined via $>$ and by (2.4) $\Delta^{J} \subset A(E)$ if and only if there exists $e \in A(E)$ such that $e>j$ for all $j$ in $J$. So the set of attractors is determined by $>$ as well.

5. Corollary. If $a_{i j}$ is a nondegenerate antisymmetric matrix and $I=\{1, \ldots, n\}$ then an ordered partition $\left(I_{0}, I_{+}, I_{-}\right)$of $I$ is called an admissible triple for $a_{i j}$ if there 
exists an equilibrium $e$ for $a_{i j}$ with $I_{\alpha}(e)=I_{\alpha}$ for $\alpha=0,+,-$. The set of admissible triples determines the relation $>$ among the equilibria and so determines the set of attractors. The set of admissible triples depends only on the component of $\left(a_{i j}\right)$ in the set of nondegenerate antisymmetric matrices (an open subset of the vector space of antisymmetric matrices). In particular, if two such matrices can be connected by a path of such matrices then they have the same set of attractors and in particular the same minimal attractor $A\left(E_{M}\right)$.

Proof: Because $e_{1}>e_{2}$ means $I_{0}\left(e_{1}\right) \cup I_{+}\left(e_{1}\right) \supset I_{0}\left(e_{2}\right)$ the relation $>$ is determined by the set of triples.

That $\left(I_{0}, I_{+}, I_{-}\right)$is an admissible for $a_{i j}$ means that $I_{0}$ is odd and the, unique up to positive multiple, solution of $\sum_{j \in I_{0}} a_{i j} x_{j}=0$ for all $i$ in $I_{0}$ is a positive vector. When normalized by $\sum_{j} x_{j}=1$ the solution is a continuous function of the matrix. So the solution for nearby matrices will also be positive. By continuity the additional conditions $a_{i x}>0$ for $i \in I_{+}$and $a_{i x}<0$ for $i \in I_{-}$will also be preserved for nearby $\left(a_{i j}\right)$.

Thus, if we call two nondegenerate antisymmetric matrices equivalent when they have the same set of admissible triples the previous paragraph shows that the equivalence classes are open sets. Because the complement, in the nondegenerate set, of an equivalence class is the union of the remaining equivalence classes, each equivalence class is relatively closed as well. Any open and closed subset is a union of components.

Q.E.D.

In the paper-rock-scissors example, $E_{M}$ consists of the vertices and $A\left(E_{M}\right)=\partial \Delta$. In the expanded example, $E_{M}$ again consists of the vertices but $A\left(E_{M}\right)=\Delta^{\{0,1,2\}} \cup \Delta^{\{0,1,3\}} \cup \Delta^{\{2,3\}}$. While the recurrence structure described above is independent of the choice of $\tau>0$ in the family $\tau a_{i j}$ by Corollary 5 , we have seen that the exact location of $\omega(p)$ in $A\left(E_{M}\right)$ for $p$ near $A\left(E_{M}\right)$ may depend on $\tau$.

In these examples, the set of minimal equilibria consists of vertices alone. This need not be true. For example, if $a_{i j}$ is given by

$$
\left(\begin{array}{ccccc}
0 & -a & -a & -a & 3 a \\
a & 0 & b & -b & -a \\
a & -b & 0 & b & -a \\
a & b & -b & 0 & -a \\
-3 a & a & a & a & 0
\end{array}\right), \quad 0<a, b<\frac{1}{3}
$$

we leave it as an exercise for the reader to show that $A\left(E_{M}\right)=\Delta^{\{0,1,2,3\}} \cup \Delta^{\{1,2,3,4\}} \cup$ $\Delta^{\{0,4\}}$ which contains, in addition to the vertices, the equilibrium $\left(0, \frac{1}{3}, \frac{1}{3}, \frac{1}{3}, 0\right)$.

As we mentioned the nice attractor structure came from the fact that $\alpha(p)$ is always an equilibrium. Because $\omega(p)$ is usually more complicated the dual repellor structure is less satisfactory. What we can say is summarized in:

6. Theorem. (a) If $p \in \Delta$, define $E(\omega(p))$ to be the set of equilibria contained in the closed invariant set $\omega(p) . e_{1}, e_{2} \in E(\omega(p))$ implies $e_{1} \approx e_{2}$.

(b) A set $\tilde{E}$ of equilibria is called initial if $e_{1}>e_{2}$ and $e_{2} \in \tilde{E}$ implies $e_{1} \in \tilde{E}$. A set $\tilde{E}$ of equilibria is initial if and only if the complementary set $E$ of equilibria is 
terminal. In that case

$$
R(\tilde{E})=\{p \in \Delta: E(\omega(p)) \subset \tilde{E}\}
$$

is the repellor dual to the attractor $A(E)$.

(c) Let $Z$ be an $\approx$ equivalence class of equilibria.

$$
B(Z)=\{p \in \Delta: \alpha(p) \in Z \text { and } E(\omega(p)) \subset Z\}
$$

is the basic set of points $\approx$ to the equilibria of $Z$. Every chain recurrent point is in some basic set and different basic sets are disjoint.

Proof: (a) follows from the fact that $\omega(p)$ is always a chain recurrent set.

Clearly $\tilde{E}$ is initial if and only if the complementary set $E$ is terminal. Let $R$ be the dual repellor for $A(E)$. We prove that $R=R(\tilde{E})$. If $p \in A(E)$ or if $p \in \Delta-(R \cup A(E))$ then $\omega(p) \subset A(E)$. So $p \in R(\tilde{E})$ implies $p \in R$. Conversely, if $p \in R$ then $\omega(p) \subset R$ because $R$ is invariant and so $\omega(p) \cap A(E)=\varnothing$. Hence, $E(\omega(p)) \cap E=\emptyset$ and so $p \in R(\tilde{E})$.

For (c) note that if $e_{+}=\alpha(p)$ and $e \in \omega(p)$ then $e_{+} \gg p \gg F(p) \gg e$. So if $e_{+} \approx e$ then $p$ is chain recurrent. Conversely, if $p$ is chain recurrent then the set $\left\{F^{n}(p): n \in \mathbb{Z}\right\}$ is a chain recurrent set and so its closure, which includes $\alpha(p)$ and $\omega(p)$, is a chain recurrent set. Thus, $p$ is a chain recurrent point if and only if $\alpha(p)$ and $E(\omega(p))$ are contained in a single $\approx$ equivalence class. Notice that if $E=\left\{e_{1}: e \gg e_{1}\right.$ for $e$ in $\left.Z\right\}$ and $\tilde{E}=\left\{e_{1}: e_{1} \gg e\right.$ for $e$ in $\left.Z\right\}$ then $\tilde{E}$ is initial and $E$ is terminal although instead of being disjoint $E \cap \tilde{E}=Z . B(Z)=R(\tilde{E}) \cap A(E)$.

Q.E.D.

Remark: By Theorem $4, A\left(E_{M}\right)$ is a basic set. It is the only basic set which is an attractor.

The definition of the dual repellors does not allow us to describe them. In particular, we leave unproved the following:

7. Conjecture. If $\tilde{E}$ is the set of equilibria complementary to the set of minimal equilibria, then the dual repellor $R(\tilde{E})$ to the attractor $A\left(E_{M}\right)$ has empty interior with respect to $\Delta$.

The set of points $p \in \Delta$ such that $\omega(p) \subset A\left(E_{M}\right)$ is precisely $\Delta-R(\tilde{E})$. This set is open because $R(\tilde{E})$ is closed. The conjecture says that it is dense as well. So if the conjecture is true then for "almost all" interior points the solution paths approach $A\left(E_{M}\right)$ as $t \in \mathbb{Z}$ approaches $\infty$. If the conjecture is false there is an open set in $\AA$ such that $F^{t}(p)$ remains bounded away from $A\left(E_{M}\right)$ although every interior point can be connected by an $\varepsilon$-chain to points of $A\left(E_{M}\right)$ for every $\varepsilon>0$.

For example, in the modified paper-rock-scissors example, $R(\tilde{E})$ is the onedimensional stable manifold for the equilibrium $e_{+}=\left(\frac{4}{7}, 0, \frac{1}{7}, \frac{2}{7}\right)$. This is a smooth invariant curve connecting $e_{-}$with $e_{+}$.

The problem with the conjecture is illustrated by Fig. 2. There we have a degenerate equilibrium for a differential equation in the plane. Instead of a one-dimensional stable manifold we have a fat inset of points approaching the origin. In our case all of the equilibria are actually hyperbolic for $F$ and so do not cause this kind of problem. However, $R(\tilde{E})$ consists not only of the stable 


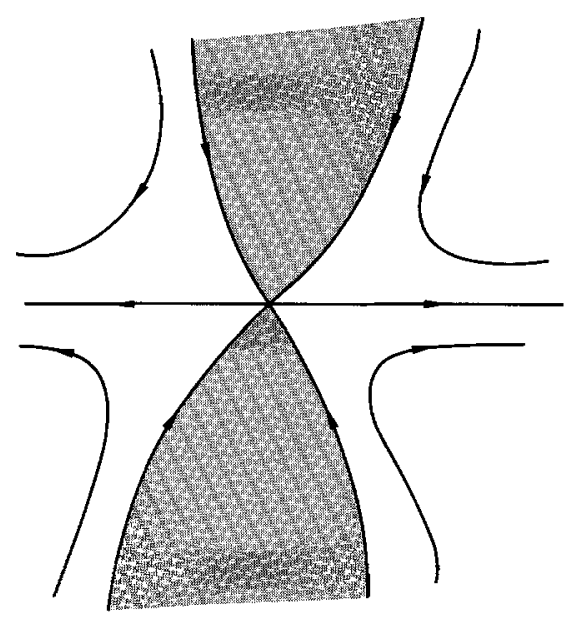

Fig. 2

manifolds for the individual equilibria, i.e. points $p$ such that $\omega(p) \in \tilde{E}$, but of the insets for the general basic sets $B(Z)$ for $Z \neq E_{M}$. Just as avoiding the problem of Fig. 2 is usually accomplished by showing that the equilibrium is hyperbolic, a proof of the conjecture in general would appear to require a hyperbolic structure on the basic sets.

Notice that in our examples, $E_{M}$ included all of the vertices. In general, if any vertex is not in $E_{M}$ the corresponding coordinate is certainly eliminated.

8. Theorem. Suppose $J_{1} \cup J_{2}=I$ and $J_{1} \cap J_{2}=\varnothing$. Suppose

$$
a_{i j}<0 \text { for all } i \in J_{1} \text { and } j \in J_{2} \text {, }
$$

i.e. $i>j$ for all $i \in J_{1}$ and $j \in J_{2}$. Define $p_{J_{1}}=\sum\left\{p_{i}: i \in J_{1}\right\}$. If $p_{J_{1}}<1$ then $\lim _{t \rightarrow \infty} F^{t}(p)_{J_{1}}=0$ i.e. all indices $i \in J_{1}$ are eliminated. In particular, $A\left(E_{M}\right) \subset \Delta^{J_{2}}$ and no vertex of $E_{M}$ is in $J_{1}$. Conversely, if $J_{1}=\left\{i \in I: i \notin E_{M}\right\}$ and $J_{2}=I-J_{1}$, then $J_{1}$ and $J_{2}$ satisfy (2.7).

Proof: Directly from the definition:

$$
\begin{aligned}
\delta p_{J_{1}} & =\sum\left\{p_{i} p_{j} a_{i j}: i \in J_{1} \text { and } j \in I\right\} \\
& =\sum\left\{p_{i} p_{j} a_{i j}: i \in J_{1} \text { and } j \in J_{2}\right\}
\end{aligned}
$$

because $\sum\left\{p_{i} p_{j} a_{i j}: i, j \in J_{1}\right\}=0$ by antisymmetry. So if $p_{J_{1}}$ is neither 0 nor 1 , i.e. there exist $i \in J_{1}$ and $j \in J_{2}$ with $p_{i}>0$ and $p_{j}>0$, then $\delta p_{J_{1}}<0$. By the usual application of Lemma 1.3(b) the decreasing sequence $F^{t}(p)_{J_{1}}$ has limit 0 as $t \rightarrow+\infty$ and 1 as $t \rightarrow-\infty$.

(d) $\Leftrightarrow(\mathrm{c})$ is obvious.

If (d) is true then by Theorem 8 the vertex at $i_{0}$ is an attractor with $i_{0}=\omega(p)$ for all $p$ such that $p_{i_{0}}>0$. This implies the last paragraph of the theorem and incidentally shows $(d) \Rightarrow(b)$.

Q.E.D.

Remark: (2.8) is true for the continuous system (1.1) when $\delta p_{J_{1}}$ is replaced by $d p_{J_{1}} / d t$. So the limit results of Theorem 8 and its corollary are true for the continuous case as well. 
Hence $\Delta^{J_{2}}$ is an attractor (let $Q=\left\{p \in \Delta: p_{J_{1}} \leqslant \frac{1}{2}\right\}$ for (1) in Smale's lemma) and so contains $A\left(E_{M}\right)$, the minimal attractor.

Conversely, if $J_{1}=\left\{i \notin E_{M}\right\}$ then because any two vertices are comparable, $i \in J_{1}$ and $j \notin J_{1}$ implies $i>j$.

Q.E.D.

In particular, we have the special case dealt with by Nagylaki:

9. Corollary. The following conditions are equivalent:

(a) $E_{M}$ contains a single equilibrium.

(b) $A\left(E_{M}\right)$ consists of a single point.

(c) $\Delta$ has no interior equilibrium and with $J=I, e_{-}$is a vertex.

(d) There exists $i_{0} \in I$ such that $a_{i_{0} j}>0$ for all $j \neq i_{0}$.

If these conditions hold and $p_{i_{0}}>0$ then $\lim _{t \rightarrow \infty} F^{t}(p)_{i_{0}}=1$. In particular, $\omega(p)$ is the vertex associated with $i_{0}$ for every interior point $p$.

Proof: $(\mathrm{b}) \Rightarrow(\mathrm{a})$ is obvious.

(a) $\Rightarrow$ (d) because if $E_{M}=\{e\}$ and $i_{0} \in \operatorname{supp}(e)$ then $e>i_{0}$ and $i_{0} \in E_{M}$ thus implies $i_{0}=e$. Because all vertices are comparable and $i_{0}$ is the unique minimal, $j>i_{0}$ for all $j \neq i_{0}$. This is (d).

\section{Hamiltonians for the continuous system}

Associated with a matrix $a_{i j}$ is the linear map and its dual which we will write as $[a]$. So in our previous notations:

$$
\begin{aligned}
& ([a] x)_{i}=a_{i x}=\sum_{j} x_{j} a_{i j} \\
& (x[a])_{j}=a_{x j}=\sum_{i} x_{i} a_{i j}
\end{aligned} \quad x \in \mathbb{R}^{n} .
$$

The condition that $e$ be an interior equilibrium for (1.1) simply says, in addition to $e \in \AA$, that $e$ is in the annihilator or kernel of the linear map [a], i.e. $[a] e=0$. If $\mathbb{R}_{0}^{n}=\left\{x \in \mathbb{R}^{n}: \sum_{i} x_{i}=0\right\}$, the perpendicular complement to the vector $\mathbf{1}=(1,1, \ldots, 1)$ in $\mathbb{R}^{n}$, then we define the subspace $B$ to be the intersection of the annihilator with $\mathbb{R}_{0}^{n}$ :

$$
B=\left\{x \in \mathbb{R}_{0}^{n}:[a] x=0\right\} .
$$

In this section we will prove that systems (1.1) admitting interior equilibria are Hamiltonian on $\dot{\Delta}$ and will in the process examine the invariant manifolds for the system. More generally, we will assume that $B$ is not the entire annihilator. This is equivalent to:

1. Assumption: There is a unique vector $q$ in $\mathbb{R}^{n}$ such that $\mathbf{1} \cdot q=1,[a] q=0$ and $b \in B$ implies $b \cdot q=0$, where $b \cdot q \equiv \sum_{i} b_{i} q_{i}$.

In other words, $q$ is the vector generating the perpendicular complement of $B$ in the annihilator, normalized by $\sum_{i} q_{i}=1$. In general, $q$ need not be a nonnegative vector even when interior equilibria exist.

Define $A$ to be the perpendicular complement of $B$ in $\mathbb{R}_{0}^{n}$ :

$$
A=\left\{x \in \mathbb{R}_{0}^{n}: b \cdot x=0 \text { for all } b \in B\right\} .
$$

Notice that $\mathbb{R}_{0}^{n}$ is the subspace of vectors tangent to our state space $\AA$. 
The vectors of $B$ yield invariant functions for the dynamical system. Recall that for $b \in \mathbb{R}^{n}$ we define the function $L^{b}(p)=\sum b_{i} \ln p_{i}$ on $\dot{\Delta}$. Lemma 1.1(a) says that for $b \in B, d L^{b} / d t$ is identically zero. Let us rewrite this result in the notation of vector fields and differential forms.

The vector field $X^{a}: \Delta \rightarrow \mathbb{R}_{0}^{n}$ associated with (1.1) is defined by

$$
X_{i}^{a}=p_{i} a_{i p} \text { at } p \in \Delta .
$$

We can compute, as we did for (1.9):

$$
d L^{b}\left(X^{a}\right)=a_{b p}=b[a] p \quad \text { at } p \in \ddot{\Delta}
$$

where the latter equation uses our new notation.

In particular, $b \in B$ implies $b[a]=-[a] b=0$ and so

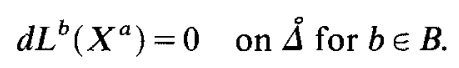

Thus, the functions $L^{b}$ slice $\triangle$ into separate invariant manifolds (a foliation of $\stackrel{\Delta}{)}$. To exhibit this structure we define for $z \in \mathbb{R}^{n}$ the linear map $E^{z}: \Delta \rightarrow \mathbb{R}$ by

$$
E^{z}(p)=p \cdot z=\sum_{i} z_{i} p_{i} .
$$

Now choose bases $\left\{b^{1}, \ldots, b^{r}\right\}$ and $\left\{z^{1}, \ldots, z^{s}\right\}$ for $B$ and $A$ respectively. Notice that $r+s=n-1$ because $A$ and $B$ form an orthogonal decomposition for $\mathbb{R}_{0}^{n}$. Define $L^{B}: \stackrel{\Delta}{\Delta} \rightarrow \mathbb{R}^{r}$ and $E^{A}: \stackrel{\Delta}{\Delta} \rightarrow \mathbb{R}^{s}$ by

$$
\begin{aligned}
& L^{B}(p)=\left(L^{b^{1}}(p), \ldots, L^{b^{r}}(p)\right) \\
& E^{A}(p)=\left(E^{z^{1}}(p), \ldots, E^{z^{s}}(p)\right) .
\end{aligned}
$$

2. Theorem. (a) The image of $E^{A}$ is an open convex set $\subseteq$ in $\mathbb{R}^{s}$ and $E^{A} \times L^{B}: \Delta \rightarrow \Delta$ $\subseteq \times \mathbb{R}^{r}$ is a diffeomorphism, i.e., it is one-to-one and onto with a smooth inverse.

(b) Fixing a vector $k$ in $\mathbb{R}^{r}$ we get a smooth s-dimensional manifold $\left(E^{A} \times\right.$ $\left.L^{B}\right)^{-1} \subseteq \times k$ on which every function $L^{b}$ for $b \in B$ is constant. Varying $k$ yields an r-parameter family of manifolds called the horizontal foliation and denoted $\mathscr{T}^{A}$. Each point $p$ of $\dot{\Delta}$ lies in a unique leaf of the foliation $\overline{\mathscr{T}}_{p}^{A}$ defined by $k=L^{B}(p)$. The leaves of $\overline{\mathscr{T}}^{A}$ are invariant manifolds for (1.1), i.e. $X^{a}$ is tangent to $\overline{\mathscr{T}}_{p}^{A}$ at $p$

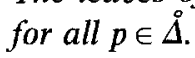

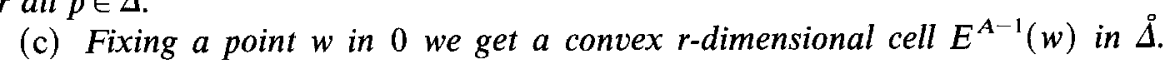
Varying $w$ yields an s-parameter family of manifolds called the vertical foliation and denoted $\overline{\mathscr{D}}^{B}$. Each point $p$ of $\dot{\Delta}$ lies in a unique leaf $\bar{D}_{p}^{B}$ defined by $w=E^{A}(p)$. Points $p_{1}, p_{2}$ in $\dot{\Delta}$ lie in the same leaf of $\overline{\mathscr{D}}^{B}$ if and only if the difference vector $p_{1}-p_{2}$ is in $B$.

(d) For $b, z \in \mathbb{R}^{n}$ define the vector fields $\bar{\nabla} L^{b}$ and $\bar{\nabla} E^{z}$ on $\dot{\Delta}$ by:

$$
\begin{aligned}
& \left(\bar{\nabla} L^{b}\right)_{i}=b_{i}-(1 \cdot b) p_{i} \\
& \left(\bar{\nabla} E^{z}\right)_{i}=p_{i}\left(z_{i}-(p \cdot z)\right)
\end{aligned} \quad \text { at } p \in \dot{\Delta}
$$

where $\mathbf{1} \cdot b=\sum_{i} b_{i}$ and $p \cdot z=\sum_{i} p_{i} z_{i}=E^{z}(p)$. We then have

$$
\begin{aligned}
d L^{b}\left(\bar{\nabla} E^{z}\right) & =d E^{z}\left(\bar{\nabla} L^{b}\right) \\
& =b \cdot z-(\mathbf{1} \cdot b)(p \cdot z) . \quad \text { at } p \in \dot{\Delta}
\end{aligned}
$$


In particular,

$$
d L^{b}\left(\bar{\nabla} E^{z}\right)=d E^{z}\left(\bar{\nabla} L^{b}\right)=0 \quad \text { if } b \in B, z \in A .
$$

So for $z \in A$ the vector field $\bar{\nabla} E^{z}$ at $p$ is tangent to the leaf $\overline{\mathscr{T}}_{p}^{A}$. Conversely, if $Y$ is a vector tangent to $\overline{\mathscr{T}}_{p}^{A}$ at $p$ then $Y=\bar{\nabla} E^{z}$ at $p$ for some $z \in A$.

Proof: This theorem is proved in Akin (1979) page 81ff. The hard part is showing that $E^{A} \times L^{B}$ is a diffeomorphism. From this the foliation results are clear because the leaves are obtained by fixing various coordinates on $\AA$ when we think of $E^{A} \times L^{B}$ as a coordinatization. Invariance of $X^{a}$ follows from (3.3). The leaf of $\overline{\mathscr{D}}^{B}, E^{A-1}(w)$, is a convex cell because $E^{A}$ is linear. Also linearity implies $E^{A}\left(p_{1}\right)=E^{A}\left(p_{2}\right)$ if and only if $z \cdot\left(p_{1}-p_{2}\right)=0$ for all $z$ in $A$. Because $p_{1}-p_{2} \in \mathbb{R}_{0}^{n}$ this is equivalent to $p_{1}-p_{2}$ in the perpendicular complement of $A$ which is $B$.

As the notation suggests $\bar{\nabla} L^{b}$ and $\bar{\nabla} E^{z}$ are gradients with respect to a suitable Riemannian metric called the Shahshahani metric. We do not need that result and instead treat (3.6) as a definition. The equations of (3.7) are easy direct

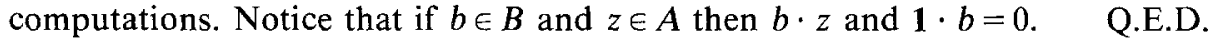

In general, a vector field is called a gradient if it is the dual of some $d f$ with respect to a Riemannian metric. $f$ is then called the associated potential function. The potential function increases on all nonequilibrium solution paths. The Shahshahani metric was originally introduced to show that the game dynamic $(0.3)$ is a gradient field with potential $(1 / 2) a_{p p}$ when the matrix $a_{i j}$ is symmetric.

A vector field is called Hamiltonian if it is the dual of some $d f$ with respect to a symplectic form, i.e. a nondegenerate exterior two-form. $f$ is called the associated Hamiltonian function. The Hamiltonian function is then conserved on all solution paths. What we will show is that when $a_{i j}$ is antisymmetric and assumption 1 holds then the restriction of $X^{a}$ to each invariant manifold $\overline{\mathscr{T}}_{p}^{A}$ is Hamiltonian with Hamiltonian function $-L^{q}$. The necessary two-form is constructed using an antisymmetric matrix related to $a_{i j}$.

3. Proposition. There exists an antisymmetric matrix $u_{i j}$ whose associated linear map $[u]$ has image $A$. Furthermore, for all $z \in A[u a] z=z$ and $[a u] z=z-(q \cdot z) \mathbf{1}$, where $[u a]$ and $[a u]$ are the linear maps associated with the product matrices $(u a)_{i j}=\sum_{k} u_{i k} a_{k j}$ and $(a u)_{i j}=\sum a_{i k} u_{k j}$.

Proof: Recall that for any antisymmetric operator the image and the kernel are perpendicular complements. In particular, the restriction to the image is nonsingular. So we can define a partial inverse operator by inverting on the image and mapping the kernel still to zero. Composing the new operator with the old one in either order we get a map which is zero on the kernel and the identity on the image, i.e. the orthogonal projection on the image. The new operator is antisymmetric because the old one was and so its matrix is antisymmetric.

We apply all this not to $a_{i j}$ but to $\tilde{a}_{i j}$ defined by

$$
\tilde{a}_{i j}=a_{i j}-n^{-1} a_{i 1}+n^{-1} a_{j 1}
$$

where $\mathbf{1}=(1,1, \ldots, 1)$ in $\mathbb{R}^{n}$.

It is easy to check that $\tilde{a}_{i j}$ is antisymmetric and $\tilde{a}_{i 1}=0$ for all $i$. Now if $b \in \mathbb{R}_{0}^{n}$

$$
\tilde{a}_{i b}=a_{i b}+n^{-1} a_{b 1}=a_{i b}-n^{-1} a_{1 b} \text {. }
$$


So if $b$ is in $B, \tilde{a}_{i b}=0$ for all $i$. Conversely, if $\tilde{a}_{i b}=0$ for all $i$ then $a_{i b}=n^{-1} a_{1 b}$. Multiply by $q_{i}$ from assumption 1 and sum on $i$ : $a_{q b}=-a_{b q}=0$ and so $n^{-1} a_{1 b}=0$ and $a_{i b}=0$ for all $i$, i.e. $b \in B$.

Thus, the kernel of $[\tilde{a}]$ is spanned by 1 and the vectors in $B$. The image of $[\tilde{a}]$ is therefore perpendicular to 1 and so lies in $\mathbb{R}_{0}^{n}$. There it is the perpendicular complement of $B$ which is $A$.

As the image of $[\tilde{a}]$ is $A$ we can apply the results of the first paragraph to define the partial inverse operator $[u]$ with antisymmetric matrix $u_{i j}$. The kernel of $[u]$ is spanned by 1 and the vectors of $B$ i.e.

$$
[u] 1=0 \quad \text { and } \quad[u] b=0 \quad \text { for } b \in B .
$$

Furthermore, for $z \in A$ we have $[u \tilde{a}] z=[\tilde{a} u] z=z$.

Now $[\tilde{a}] z$ differs from $[a] z$ by a multiple of 1 because $z$ is in $A \subset \mathbb{R}_{0}^{n}$. So $[u] \mathbf{1}=0$ implies $[u a] z=[u][a] z=[u][\tilde{a}] z=[u \tilde{a}] z=z$.

To compute $[a u] z$ we apply $[\tilde{a}]$ to the vector $q$ of assumption 1:

$$
\tilde{a}_{i q}=a_{i q}-n^{-1} a_{i 1}+n^{-1} a_{q 1}=-n^{-1} a_{i 1}
$$

because $a_{i j}$ annihilates $q$, i.e. from (3.8):

$$
a_{i j}=\tilde{a}_{i j}-\tilde{a}_{i q}+\tilde{a}_{j q}
$$

and

$$
\begin{aligned}
(a u)_{i j} & =(\tilde{a} u)_{i j}+\sum_{k}\left(-\tilde{a}_{i q}+\tilde{a}_{k q}\right) u_{k j} \\
& =(\tilde{a} u)_{i j}-\sum_{k} u_{j k} \tilde{a}_{k q}=(\tilde{a} u)_{i j}-q_{j}+n^{-1}
\end{aligned}
$$

where we have used $\sum_{k} u_{k j}=0$ by (3.9) and $[u \tilde{a}](q)=$ the perpendicular projection of $q$ to $A$ which is $q-n^{-1} 1$ because $q$ is perpendicular to $B$.

Hence, for $z \in A \subset \mathbb{R}_{0}^{n}$

$$
([a u] z)_{i}=([\tilde{a} u] z)_{i}-q \cdot z=z_{i}-q \cdot z .
$$

What remains is an exercise in the use of differential forms. We follow the exterior algebra conventions of Abraham and Marsden (1978). Define on $\Delta$ the two-form:

$$
\begin{aligned}
\omega & =\frac{1}{2} \sum_{i, j} \frac{u_{j i}}{p_{i} p_{j}} d p_{i} \wedge d p_{j} \\
& =\frac{1}{2} \sum_{i, j} u_{j i} d \ln p_{i} \wedge d \ln p_{j} \\
& =d\left[\frac{1}{2} \sum_{i, j} u_{j i} \ln p_{i} d \ln p_{j}\right] \quad \text { at } p \in \dot{\Delta} .
\end{aligned}
$$

The third version shows that $\omega$ is exact and so is closed.

Recall that if $\omega$ is a two-form and $X$ is a vector field then the inner product of $X$ and $\omega$, denoted $i_{X} \omega$ is the one-form defined by $i_{X} \omega(Y)=\omega(X, Y)$. For example using $X=\bar{\nabla} E^{z}$ we compute:

$$
i_{\bar{\nabla} E^{z}} \omega=-d L^{z[u]}
$$


as follows:

$$
\begin{aligned}
\omega\left(\bar{\nabla} E^{z}, Y\right) & =\frac{1}{2} \sum_{i, j} u_{j i}\left[d \ln p_{i}\left(\bar{\nabla} E^{z}\right) d \ln p_{j}(Y)-d \ln p_{i}(Y) d \ln p_{j}\left(\bar{\nabla} E^{z}\right)\right] \\
& =\sum_{i, j} u_{j i}\left(z_{i}-(p \cdot z)\right) d \ln p_{j}(Y) \quad\left(\text { by antisymmetry of } u_{i j}\right) \\
& =\sum_{j}([u] z)_{j} d \ln p_{j}(Y) \quad\left(\text { because } \sum_{i} u_{j i}=0\right) \\
& =-d\left(\sum_{j}(z[u])_{j} \ln p_{j}\right)(Y) .
\end{aligned}
$$

4. Theorem. The restriction of $\omega$ to each leaf of $\overline{\mathscr{T}}^{A}$ is a symplectic form, i.e. a closed, nondegenerate two-form. Suppose $q \in \mathbb{R}^{n}$ such that $\mathbf{1} \cdot q=1$ and $[a] q=0$, e.g. the vector $q$ of assumption 1 . On each leaf of $\overline{\mathscr{T}}^{A}$ the vector field $X^{a}$ is $\omega$-dual to $d\left(-L^{q}\right)$, i.e. for any vector $Y$ tangent to $\overline{\mathscr{T}}_{p}^{A}$ at $p$ :

$$
i_{X^{a}} \omega(Y)=d\left(-L^{q}\right)(Y) .
$$

Proof: Fix $p \in \dot{\Delta}$ and recall that evaluating the vector fields $\bar{\nabla} E^{z}$ at $p$ as $z$ varies over $A$ we get all of the vectors tangent to $\overline{\mathscr{T}}_{p}^{A}$.

Now suppose $z, \tilde{z} \in A$ and apply (3.11) and then (3.7) with $b=z[u]$ to get

$$
\begin{aligned}
\omega\left(\bar{\nabla} E^{z}, \bar{\nabla} E^{\tilde{z}}\right) & =i_{\bar{\nabla} E^{z}} \omega\left(\bar{\nabla} E^{\tilde{z}}\right) \\
& =-d L^{z[u]}\left(\bar{\nabla} E^{\tilde{z}}\right)=-z[u] \tilde{z}
\end{aligned}
$$

because $1 \cdot z[u]=z[u] 1=0$.

Recall that $[u]$ is an antisymmetric operator with image $A$. So $-z[u] \tilde{z}=0$ for all $\tilde{z}$ in $A$ implies $0=-z[u]=[u] z$ and so $z=0$ because $[u]$ is nonsingular on $A$.

This means that if $Y$ is a vector tangent to $\overline{\mathscr{T}}_{p}^{A}$ at $p$, and so $Y=\bar{\nabla} E^{z}$ for some $z$, and $i_{Y} \omega$ vanishes at all vectors tangent to $\overline{\mathscr{T}}_{p}^{A}$ at $p$ then $Y=0$. This is what it means for $\omega$ to be nondegenerate on $\overline{\mathscr{T}}_{p}^{A}$. Thus, we have that the restriction of $\omega$ to $\overline{\mathscr{T}}_{p}^{A}$ is a symplectic form.

Similarly, we need only prove Eq. (3.12) with $Y=\bar{\nabla} E^{z}$ for $z \in A$. Assume first that $q$ is the vector of assumption 1. Apply (3.11) and (3.2)

$$
\begin{aligned}
i_{X^{a} \omega}\left(\bar{\nabla} E^{z}\right) & =-i_{\bar{\nabla} E^{z}} \omega\left(X^{a}\right) \\
& =d L^{z[u]}\left(X^{a}\right)=z[u][a] p=z[u a] p .
\end{aligned}
$$

Because $u_{i j}$ and $a_{i j}$ are both antisymmetric the transpose of $u a$ is $a u$ and so $z[u a] p=p[a u] z$. By Proposition 3, $[a u] z=z-(q \cdot z) 1$ and so we get

$$
i_{X^{a} \omega}\left(\bar{\nabla} E^{z}\right)=(p \cdot z)-(q \cdot z) .
$$

On the other hand, from (3.7) and $1 \cdot q=1$ we get:

$$
d L^{q}\left(\bar{\nabla} E^{z}\right)=(q \cdot z)-(p \cdot z) .
$$

This proves (3.12) with our special choice of vector $q$. In general, if $\mathbf{1} \cdot \tilde{q}=1$ and $[a] \tilde{q}=0$ then the difference $b=\tilde{q}-q$ lies in $B$ and so $L^{\tilde{q}}=L^{q}+L^{b}$. Because $d L^{b}(Y)=0$ for all vectors $Y$ tangent to $\overline{\mathscr{T}}_{p}^{A}(3.12)$ holds with $q$ replaced by $\tilde{q}$. 
If $e$ is an interior equilibrium then $1 \cdot e=1$ and $[a] e=0$. Recall that the function $I^{e}$ defined by (1.8) differs from $-L^{e}$ by a constant. We immediately get the following:

5. Corollary. If $E_{0}$, the set of interior equilibria, is nonempty then on each leaf of $\overline{\mathscr{T}}^{A}$ the vector field $X^{a}$ is $\omega$-dual to $d I^{e}$ for all $e \in E_{0}$.

Note that if $e \in E_{0}$ and $p \in \dot{\Delta}$ then $p \in E_{0}$ if and only if the difference vector $p-e$ lies in $B$, i.e. if $[a] e=0$ then $[a] p=0$ if and only if $[a](p-e)=0$. So by Theorem $2(\mathrm{c}), E_{0}$ is either empty or is exactly a single leaf of the vertical foliation $\overline{\mathscr{D}}^{B}$. So every horizontal leaf intersects $E_{0}$ in exactly one point and we can define

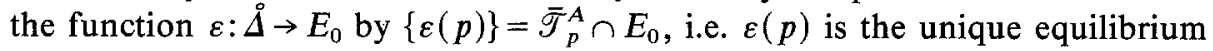
with the same horizontal leaf as $p$. $\varepsilon$ is a smooth function because if $e$ is any fixed point of $E_{0}: \varepsilon(p)=\left(E^{A} \times L^{B}\right)^{-1}\left(E^{A}(e), L^{B}(p)\right)$. Using $\varepsilon(p)$ we can define

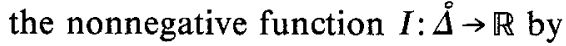

$$
I(p)=I^{\varepsilon(p)}(p)=-\sum_{i} \varepsilon(p)_{i} \ln \left(p_{i} / \varepsilon(p)_{i}\right) .
$$

Given any $e \in E_{0}$, on the horizontal leaf $\overline{\mathscr{T}}_{e}^{A} \varepsilon(p)=e$ is constant and so as $p$ varies in $\overline{\mathscr{T}}_{e}^{A}, I(p)=I^{e}(p)$. So $I \geqslant 0$ and vanishes, in the leaf, only at $e$. Consequently, $I=0$ precisely on the set $E_{0}$. Because $I=I^{e}$ on $\tilde{T}_{e}^{A}, X^{a}$ is $\omega$-dual to $d I$ on each horizontal leaf.

The Hamiltonian function associated with a Hamiltonian vector field is unique up to additive constant given the symplectic form. Notice that the different candidates we have given for the Hamiltonian: $-L^{q}, I^{e}, I$ do differ by an additive constant on any fixed horizontal leaf. That this constant may differ from leaf to leaf is a reminder of the fact that equation of duality (3.12) is only true for vectors tangent to the leaves.

While these results are all restricted to the leaves we can use them to construct

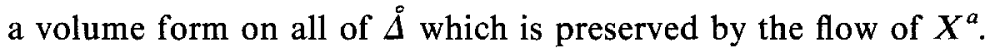

6. Theorem. $s=$ dimension $A$ is even and $r=$ dimension of $B$ is $n-1-s$. Choose $\left\{b^{1}, \ldots, b^{r}\right\}$ a basis for $B$ and define

$$
\Omega=\omega^{s / 2} \wedge d L^{b^{1}} \wedge \cdots \wedge d L^{b^{r}}
$$

where $\omega^{s / 2}$ is the $s$-form $\omega \wedge \omega \cdots \wedge \omega$ (s/2 times). $\Omega$ is a volume form on $\dot{\Delta}$, i.e. a nonvanishing $n-1$ form, and $\Omega$ is invariant for the flow associated with $X^{a}$.

Proof: We will just sketch the argument which is a technical application of the Lie derivative operator $L_{X^{a}}$ associated with the vector field $X^{a}$. For simplicity we will assume $\left\{b^{1}, \ldots, b^{r}\right\}$ is an orthonormal basis for $B$, i.e. $b^{k} \cdot b^{l}=1$ if $k=l$ and $=0$ if $k \neq l$. Changing the basis just multiplies $\Omega$ by a nonzero constant.

Because each function $L^{b}$ with $b$ in $B$ is invariant for $X^{a}$, the Lie derivative $L_{X^{a}} d L^{b}=0$ for $b \in B$. So:

$$
L_{X^{a}} \Omega=L_{X^{a}}\left(\omega^{s / 2}\right) \wedge d L^{b^{1}} \wedge \cdots \wedge d L^{b^{r}} .
$$

Because $L_{X^{a} \omega_{1}} \wedge \omega_{2}=\left(L_{x}{ }^{a} \omega_{1}\right) \wedge \omega_{2} \pm \omega_{1} \wedge L_{X^{a} \omega_{2}}$ with the choice of sign depending on the degree of $\omega_{1}$. (Recall that the antisymmetric matrix $u_{i j}$ is nonsingular when restricted to $A$ and so $A$ has even dimension.) 
Now if $\left\{z^{1}, \ldots, z^{s}\right\}$ is a basis for $A$ then because $A$ is perpendicular to $B$ in $\mathbb{R}_{0}^{n}$ (3.7) implies

$$
d L^{b^{k}}\left(\bar{\nabla} E^{z^{l}}\right)=0, \quad k=1, \ldots, r \text { and } l=1, \ldots, s .
$$

On the other hand, because the basis for $B$ is orthonormal (3.7) also implies

$$
d L^{b^{k}}\left(\bar{\nabla} E^{b^{l}}\right)= \begin{cases}1 & k=l \\ 0 & k \neq l\end{cases}
$$

Consequently, if $\tau$ is any $s$-dimensional form

$$
\begin{aligned}
\tau & \wedge d L^{b^{1}} \wedge \cdots \wedge d L^{b^{r}}\left(\bar{\nabla} E^{z^{1}}, \ldots, \bar{\nabla} E^{z^{s}}, \bar{\nabla} E^{b^{1}}, \ldots, \bar{\nabla} E^{b^{r}}\right) \\
& =\tau\left(\bar{\nabla} E^{z^{1}}, \ldots, \bar{\nabla} E^{z^{s}}\right) .
\end{aligned}
$$

We apply this result first with $\tau=\omega^{s / 2}$ and then with $\tau=L_{X^{a}}\left(\omega^{s / 2}\right)$. Because the restriction of $\omega$ to each horizontal leaf is a symplectic form, $\omega^{s / 2}$ restricts to a volume form on each leaf and this means

$$
\omega^{s / 2}\left(\bar{\nabla} E^{z^{1}}, \ldots, \bar{\nabla} E^{z^{s}}\right) \neq 0,
$$

i.e. $\omega^{s / 2}$ does not vanish when applied to a basis for the tangent space of the leaf. (3.15) then implies that $\Omega$ never vanishes.

On the other hand, $X^{a}$ is Hamiltonian on the leaves and this means that the restriction of $L_{X^{a} \omega}$ is 0 on the leaves and hence so is the restriction of $L_{X^{a}}\left(\omega^{s / 2}\right)$. Consequently,

$$
L_{X^{a} \omega^{s / 2}}\left(\bar{\nabla} E^{z^{1}}, \ldots, \bar{\nabla} E^{z^{s}}\right)=0
$$

at every point. (3.15) and the previous computation for $L_{X^{a}} \Omega$ then imply that $L_{X^{a}} \Omega$ is identically 0 . By definition of the Lie derivative, $\Omega$ is thus an invariant form for $X^{a}$.

Q.E.D.

We conclude with some general remarks.

Because they are usually structurally unstable, Hamiltonian differential systems are only appropriate when the conservation effects are essential in the underlying theory (e.g. mechanical systems) rather than accidental consequences of the choice of model design (e.g. the original Lotka-Volteria predator-prey equations). In particular, we think that Nagylaki is absolutely correct in his preference for the discrete time model for the biological applications he has in mind. On the other hand. Hamiltonian dynamics per se is an actively growing, fruitful branch of pure mathematics. It is our hope that these simple appearing systems may provide a rich new collection of examples.

\section{References}

1. Abraham, R., Marsden, J.: Foundation of Mechanics (2nd Ed.) Reading, Mass. The Benjamin/Cummings Publishing Company, Inc. 1978

2. Akin, E.: The Geometry of Population Genetics. Lecture Notes in Biomathematics, Vol. 31. Springer 1979

3. Akin, E., Hofbauer, J.: Recurrence of the Unfit. Math. Biosciences 61, 51-62 (1982)

4. Conley, C.: Isolated Invariant Sets and the Morse Index. CBMS Conference Series No. 38, American Mathematical Society 1978 
5. Eigen, M., Schuster, P.: The Hypercycle. Springer 1979

6. Gale, D.: The Theory of Linear Economic Models. New York: McGraw-Hill 1960

7. Hines, W. G. S.: Three Characterizations of Population Strategy Stability. J. Appl. Prob. 17, 333-340 (1980)

8. Hofbauer, J.: A difference equation model for the hypercycle. SIAM J. Appl. Math. to appear (1984)

9. Karlin, S.: Mathematical Methods and Theory in Games, Programming and Economics. Reading, Mass. Addison-Wesley 1959

10. Losert, V., Akin, E.: Dynamics of games and genes: Discrete versus continuous time. J. Math. Biol. 17, 241-251 1983

11. Maynard Smith, J.: Evolution and the Theory of Games. Cambridge: Cambridge University Press 1982

12. Nagylaki, T.: Evolution of a large population under gene conversion. Proc. Natl. Acad. Sci. USA 80, 5941-5945 1983a

13. Nagylaki, T.: Evolution of a finite population under gene conversion. Proc. Natl. Acad. Sci. USA 80, 6278-6281 1983b

14. Smale, S.: The $\Omega$-stability Theorem. Proc. Symp. in Pure Math. Vol. XIV, American Mathematical Society 1970

15. Taylor, P., Jonker, L.: Evolutionarily stable strategies and game dynamics. Math. Biosci. 40, $145-1561978$

Received April 16, 1984 